\title{
Modeling Industrial Dynamics with Innovative Entrants
}

S.G. Winter

Y.M. Kaniovski

G. Dosi

RR-01-03

January 2001 


\section{Modeling Industrial Dynamics with Innovative Entrants}

\section{S.G. Winter}

The Wharton School, University of Pennsylvania, Philadelphia, USA

Y.M. Kaniovski

International Institute for Applied Systems Analysis, Laxenburg, Austria and University of Trento, Italy

G. Dosi

The Sant' Anna School of Advanced Studies, Pisa, Italy

RR-01-03

January 2001

Reprinted from Structural Change and Economic Dynamics 11 (2000) 255-293. 
Research Reports, which record research conducted at IISA, are independently reviewed before publication. Views or opinions expressed herein do not necessarily represent those of the Institute, its National Member Organizations, or other organizations supporting the work.

Reprinted with permission from Structural Change and Economic Dynamics 11 (2000) 255-293. Copyright (c) 2000 Elsevier Science B.V.

All rights reserved. No part of this publication may be reproduced or transmitted in any form or by any means, electronic or mechanical, including photocopy, recording, or any information storage or retrieval system, without permission in writing from the copyright holder. 


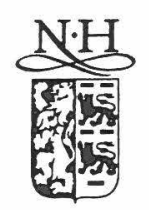

ELSEVIER

Structural Change and Economic Dynamics

11 (2000) 255-293

\title{
Modeling industrial dynamics with innovative entrants
}

\author{
S.G. Winter ${ }^{\text {a }}$, Y.M. Kaniovski ${ }^{\text {b,c }}$, G. Dosi ${ }^{\text {d,* }}$ \\ a The Wharton School, University of Pennsyliania, 2000 Steinberg Hall-Dietrich Hall, Philadelphia, \\ PA 19104.6370, USA \\ ${ }^{\mathrm{b}}$ The International Institute for Applied Systems Analysis, A-2361 Laxenburg, Austria \\ ' University' of Trento, Via Inama 5, I-38100 Trento, Italy \\ 'The Sant'Anna School of Advanced Studies, Via Carducci 40, I-56127 Pisa, Italy
}

Accepted 22 June 1999

\begin{abstract}
The paper analyzes some generic features of industrial dynamics whereby innovative change is carried, stochastically, by new entrants. Relying on the formal representation suggested in Winter, S.G., Kaniovski, Y.M., Dosi, G., 1997. A Baseline Model of Industry Evolution. [Interim Report IR-97-013/March, International Institute for Applied Systems Analysis, Laxenburg, Austria], it studies both the asymptotic properties of such processes and their appropriability to account for a few empirical stylized facts, including persistent entry and exit, skewed size distributions and turbulence in market shares. (C) 2000 Elsevier Science B.V. All rights reserved.
\end{abstract}

Keywords: Evolution; Competition; Learning; Stochastic entry; Entrepreneurial startups; Expanding set of technological opportunities; Industrial dynamics

\section{Introduction}

In this work we explore the dynamic features of industries characterized by the persistent arrival of innovative entrants. The models which follow build upon and modify the baseline model presented in Winter et al. (1997). In an extreme synthesis, in the latter we develop a framework of analysis of the competitive

\footnotetext{
* Corresponding author.

E-mail address: dosi@sssup.it (G. Dosi).
} 
dynamics of industries composed of heterogeneous firms and continuing stochastic entry. There, we show that despite the simplicity of the assumptions, the model is able to account for a rather rich set of empirical 'stylized facts', such as: (i) continuing turbulence in market shares; (ii) persistent inflows and outflows of firms; (iii) 'life cycle' phenomena - including, in particular, nearer the birth of an industry, relatively sudden 'shakeouts', yielding distinctly different industrial structure thereafter; and (iv) skewed size distributions of firms ${ }^{1}$.

The 'heroic' simplicity of Winter et al. (1997) goes as far as assuming that the set of technological options among which entrants draw - as a formal metaphor of their diverse capabilities - is given from the start and is invariant throughout the unfolding evolution of the industry. While this assumption is certainly in tune with the spirit of most evolutionary game-theoretical set-ups, it is also at odds with an overwhelming empirical evidence highlighting the role of innovators as carriers of technological and organizational discoveries. Typically, these discoveries happen to be tapped at some point in the history of an industry on the grounds of the available knowledge base at that time, but would not have been possible earlier on, given the knowledge base at that earlier time.

More formally, this implies that what is commonly called the 'production possibility set' endogenously shifts, due to the cumulative (but stochastic) effects of exploration by potential innovators ${ }^{2}$.

The model which follows studies the properties of industrial dynamics which correspond to that archetype of industrial evolution which some authors call Schumpeter Mark I regime (cf. Dosi et al., 1995; Malerba and Orsenigo, 1995). In short, while of course both incumbents and new entrants empirically attempt to explore - to varying degrees - yet unexploited opportunities of innovation, here we focus upon the properties of that extreme archetype whereby only entrants have a positive probability of advancing the current state of technological knowledge. (Hence the name of such a 'regime', in analogy with the emphasis of Schumpeter (1934) upon novel entrepreneurial efforts as drivers of change.)

Compared with the cited 'baseline model' discussed in Winter et al. (1997), in the following we shall try to disentangle those properties which appear to be generic features of a wide class of processes of industrial dynamics simply resting upon persistently heterogeneous agents and market selection and, conversely, those properties which depend upon more specific forms of innovative learning, such as the Schumpeter Mark I regime considered here ${ }^{3}$. As we shall show below, some of the emerging 'stylized facts' of the modeled dynamics appear to robustly hold in

\footnotetext{
${ }^{1}$ This evidence is discussed at much greater length in the special issues of Industrial and Corporate Change, 5, 1997 and of The International Journal of Industrial Organization, 4, 1995. See also Baldwin (1995); Carroll and Hannan (1995); Davis et al. (1996); Dunne et al. (1988); Dosi et al. (1995); Geroski (1995); Hannan and Freeman (1989).

${ }^{2}$ For more detailed empirical corroborations of these points, cf., among others, Dosi (1988) and Freeman and Soete (1997).

${ }^{3}$ See Winter et al. (1997) also for some comparative assessment of somewhat germane models of industrial dynamics based on much more stringent assumptions of individual forward-looking rationality and collective equilibrium, such as Jovanovic (1982), Hopenhayn (1992), and Ericson and Pakes (1995).
} 
both set-ups, with or without innovative entry. Other features, including some path-dependence properties, interestingly, appear only when 'openended' dynamics on technological opportunities is accounted for, as we do in this work.

Section 2 sets out the basic structure of the model, in a first specification with innovative learning by entrants directed at increasing capital productivity, and, conversely, in Section 3, we study the properties of a symmetrical assumption of (stochastically) increasing labour efficiencies.

\section{The basic framework of the model: a first setting with increasing capital efficiencies}

Let us assume an industry evolving in discrete time $t=0,1, \ldots$. At $t=0$ there are no firms ready to produce, but $k$ firms arrive to the industry, ready to start manufacturing at $t=1$. Techniques are capital-embodied and firm-specific. So, the model which follows can be interpreted as a vintage capital model, with heterogeneous techniques across firms also within each vintage.

At time $t \geq 1$ the industry consists of $n_{t}$ firms which are involved in production and a number of new firms that enter at $t$ and will participate in manufacturing from $t+1$ onward. Uniformly for the whole industry we have:

$v$ - price per unit of physical capital, $v>0$,

$d$ - depreciation rate of the capital stock, $0<d \leq 1$.

In the first version of the model which follows the output is produced by capital alone. The competitiveness of any firm represented in the industry is ultimately determined by its capital per unit of output. Let us designate the latter by $a_{i}$ for the $i$-th firm. As time goes on, the 'best' capital/output ratio (in real terms) attainable in the industry stochastically decreases.

Let us further assume the following endogenous stochastic mechanism of learning by entrants. Take a random variable $\xi$ with positive mean $E \xi$ and a finite variance $D \xi$. Set $\zeta$ for a random variable distributed over $[a, b], 0<a<b<\infty$. For each time instant $t \geq 0$ we allow for the industry to have $k \geq 1$ new firms whose levels of capital per unit of output are randomly determined as $\exp \left\{-A_{t}\right\} \zeta^{i}, t k+1 \leq i \leq$ $(t+1) k$. Here $A_{t+1}=A_{t}+\xi^{t+1}, t \geq 0, A_{0}=\xi^{0}$ Also, $\xi^{t}, t \geq 0$, and $\xi^{i}, i \geq 1$, are mutually independent collections of realizations of $\xi$ and $\zeta$. Thus, all capital ratios feasible for newcomers at time $t$ belong to $\left[\exp \left\{-A_{t}\right\} a, \exp \left\{-A_{t}\right\} b\right]$. Their distribution within this interval is governed by a realization of $\exp \left\{-A_{t}\right\} \zeta$. Consequently, $A_{t}$ characterizes in a probabilistic way the highest productivity of capital attainable to newcomers in the industry at time $t$. Note that by construction in this competitive environment only newcomers learn to improve the productivity of capital.

It is important to notice that the assumption of a fixed number of entrants is just made here for expositional simplicity. The qualitative results do not change if one 
allows stochastic entry (as we in fact do in Winter et al., 1997) and if entry probabilities were made dependent upon some state variable of the system, for example, the current level of profitability in the industry: see Remark 2.2. below.

The productive capacity of the $i$-th firm is $Q_{t}^{i}=K_{i}(t) / a_{i}$, where $K_{i}(t)$ stands for the capital of the $i$-th firm at time $t$. The total productive capacity of the industry involved in manufacturing at time $t$ is

$$
Q_{t}=\sum_{i=1}^{n_{t}} Q_{t}^{i}
$$

We assume a decreasing continuous demand function $p=H(q)$, mapping $[0, \infty)$ in $[0, H(0)]$ such that $H(0)<\infty$ and $H(q) \rightarrow 0$ as $q \rightarrow \infty$, where as usual, $p$ stands for the price and $q$ for demanded quantities. [Thus, the price at time $t$ equals $H\left(Q_{t}\right)$.] The gross profit per unit of output at $t$ is also $H\left(Q_{t}\right)$ since, without loss of generality we may also assume zero variable costs. The gross investment per unit of output at $t$ is a share of the gross profit, i.e. $\lambda H\left(Q_{t}\right)$, where the constant $\lambda$ captures the share of the gross profit which does not leak out as the interest payments and shareholders' dividends, and can be considered to be a measure for the propensity to invest. The total gross investment per unit of capital for the $i$-th firm at time $t$ reads $i H\left(Q_{t}\right) / v a_{i}$.

For each capital ratio generated at $t$ we shall allow a single entrant. Entrants' initial capitals are independent realizations $\theta^{i}, i \geq 1$, of a random variable $\theta$ distributed over $[c, h], 0<c<h<\infty$. (It is assumed that the realizations of $\xi, \zeta$ and 0 are mutually independent random variables.)

To complete the description of the competitive environment we need some death mechanism. A firm is dead at time $t$ and does not participate in the production process from $t+1$ onward if its capital at $t$ is less than $\varepsilon c, \varepsilon \in(0,1]^{4}$

We assume that all random elements are given on a probability space $\{\Omega, \mathscr{F}, P\}$.

In order to study the long run behavior of this industry, let us give a formal description of its evolution.

\subsection{A dynamical setting of the model}

Let firm $i$ be manufacturing during time $t$. Our investment rule implies that at the end of this production period its capital is

$$
Q_{t}^{i} a_{i}\left[1-d+\frac{\lambda}{v a_{i}} H\left(Q_{t}\right)\right] .
$$

If this value does not drop below the death threshold $\varepsilon c$, the firm continues to manufacture during time instant $t+1$. Otherwise it dies. To capture these possibilities, we introduce

\footnotetext{
${ }^{4}$ The situation without mortality can be thought of as a limit case when $\varepsilon=0$. Conversely, for a possible refinement of the above mortality rule see Remark 2.3 below.
} 


$$
\chi_{Q t}\left[1-d+\frac{\lambda}{v a_{i}} H\left(Q_{t}\right)\right] \geq \varepsilon c / a_{i}
$$

the indicator function of the event that the firm continues to manufacture. As usual, for a relation $\mathscr{A}$ we set that

$$
\chi \mathscr{A}=\left\{\begin{array}{l}
1, \quad \text { if } \mathscr{A} \text { is true } \\
0, \quad \text { otherwise }
\end{array}\right.
$$

Now the evolution of the $i$-th firm (in terms of productive capacity) reads

$$
Q_{t+1}^{i}=Q_{t}^{i}\left[1-d+\frac{\lambda}{v a_{i}} H\left(Q_{t}\right)\right] \chi_{Q_{t} i\left[1-d+\frac{\lambda}{v a_{i}} H\left(Q_{t}\right)\right] \geq \varepsilon c / a_{i}} .
$$

These equations are not handy for analysis. Mortality implies that $n_{t}$, the number of firms in business, changes over time. Thus, we have a system with a variable dimension. Moreover, these equations do not incorporate the entry process: hence Eq. (2.1) only captures a part of the evolution of the industry. In order to handle entry and variable numbers of incumbents one needs a dynamic representation of the model that leaves room for all feasible development paths. It is nested in an infinite dimensional space.

The intuition is the following. Even if at each time one assumes, quite naturally, a finite number of entrants, as time goes to infinity, one must allow for an infinite number of firms to visit the industry. Moreover, the number of firms is normally changing over time as the joint outcome of entry and selection (entailing mortality). Somewhat similar considerations apply to the input coefficients (i.e. the productivities) which the system explores. The rather novel formal machinery developed below is precisely aimed to rigorously capture these properties.

Introduce a space $R_{\infty}$ of vectors with denumerably many coordinates. Set

$$
R_{x}=R \otimes\left[\bigotimes_{i=1}^{\infty} R_{i}\right],
$$

where $\otimes$ stands for the direct sum of a real line $R$ and $2 k$-dimensional real vector spaces $R_{i}, i \geq 1$. Thus, for every $\mathbf{q} \in R_{\infty}$

$$
\mathbf{q}=q \otimes\left[\bigotimes_{i=1}^{\infty} \mathbf{q}^{i}\right]
$$

with $q \in R$ and $q^{i} \in R_{i}, i \geq 1$. Define an automorphism $\mathbf{D}(\cdot)$ on $R_{\infty}$ such that

$$
\mathbf{D}(\mathbf{q})=\mathbf{D}^{\mathbf{1}}(\mathbf{q}) \otimes\left[\bigotimes_{i=2}^{\infty} \mathbf{D}^{i}(\mathbf{q})\right] \text {, }
$$

where $\mathbf{D}^{1}(\cdot): R_{\infty} \mapsto R \otimes R_{1} \otimes R_{2}$ and $\mathbf{D}^{i}(\cdot): R_{\infty} \mapsto R_{i}, i \geq 3$. Let

$$
\begin{aligned}
& D_{1}^{1}(\mathbf{q})=q, \quad D_{s}^{1}(\mathbf{q})=0, \quad 2 \leq s \leq 2 k+1, \quad D_{2 k+j+1}^{1}(\mathbf{q})=q_{j}^{1} \exp \{-q\} \chi_{\alpha_{j}^{\prime}(\mathbf{q})}, \\
& D_{3 k+j+1}^{1}(\mathbf{q})=
\end{aligned}
$$




$$
\begin{aligned}
& q_{k+j}^{1}\left[1-d+\frac{\lambda}{v} H\left(\sum_{s=1}^{k}\left[q_{k+s}^{1} \exp \{q\} / q_{s}^{1}+\sum_{i=2}^{\infty} q_{k+s}^{i} / q_{s}^{i}\right]\right) \exp \{q\} / q_{j}^{1}\right] \chi_{\cdot \sigma_{j}(\mathbf{q})} ; \\
& D_{j}^{i}(\mathbf{q})=q_{j}^{i} \chi_{j_{j}^{i}(\mathbf{q})}, \\
& D_{k+j}^{i}(\mathbf{q})=q_{k+j}^{i}\left[1-d+\frac{\lambda}{v} H\left(\sum_{s=1}^{k}\left[q_{k+s}^{1} \exp \{q\} / q_{s}^{1}+\sum_{p=2}^{\infty} q_{k+s}^{p} / q_{s}^{p}\right]\right) / q_{j}^{i}\right] \chi_{\alpha_{j}^{i}(\mathbf{q})},
\end{aligned}
$$

where $1 \leq j \leq k, i \geq 2, \mathscr{A}_{j}^{1}(\mathbf{q})$ designates the relation

$$
q_{k+j}^{1}\left[1-d+\frac{\lambda}{v} H\left(\sum_{s=1}^{k}\left[q_{k+s}^{1} \exp \{q\} / q_{s}^{1}+\sum_{i=2}^{\infty} q_{k+s}^{i} s / q_{s}^{i}\right]\right) \exp \{q\} / q_{j}^{1}\right] \geq \varepsilon c
$$

and $\mathscr{A}_{j}^{i}(\mathbf{q})$ stands for the relation

$$
q_{k+j}^{i}\left[1-d+\frac{\lambda}{v} H\left(\sum_{s=1}^{k}\left[q_{k+s}^{1} \exp \{q\} / q_{s}^{1}+\sum_{p=2}^{\infty} q_{k+s}^{p} / q_{s}^{i}\right]\right) / q_{j}^{i}\right] \geq \varepsilon c .
$$

We restrict ourselves to vectors $q$ defined by Eq. (2.2) belonging to

$$
R_{\infty}^{+}=[0, \infty) \otimes\left[\bigotimes_{i=1}^{\infty} R_{i}^{+}\right]
$$

and set $H(\infty)=0$ for the case when the iterated sum involved in the above expressions is infinite. Here

$$
R_{i}^{+}=\left\{\mathbf{q}^{i} \in R_{i}: \quad q_{j}^{i}>0, q_{k+j}^{i} \geq 0, j=1,2, \ldots, k\right\}, \quad i \geq 1 .
$$

Also, $D_{s}^{i}(\cdot)$ and $q_{s}^{i}$ stand for the $s$-th coordinates of $\mathbf{D}^{i}(\cdot)$ and $\mathbf{q}^{i}$.

Define infinite dimensional random vectors $\mathbf{Y}^{t}, t \geq 0$, setting

$$
\begin{aligned}
& Y_{1}^{t}=\xi^{t}, \quad Y_{i+1}^{t}=\zeta^{i k+i}, \quad Y_{k+i+1}^{t}=\theta^{i k+i}, \quad i=1,2, \ldots, k, \quad Y_{j}^{t}=0 \\
& j \geq 2 k+2 .
\end{aligned}
$$

(Note that here we number coordinates linearly rather than in terms of cohorts as above.)

The evolution of the industry is as follows

$$
\mathbf{q}(t+1)=\mathbf{D}(\mathbf{q}(t))+\mathbf{Y}^{t+1}, \quad t \geq 0, \quad \mathbf{q}(0)=Y^{0},
$$

Since $\mathbf{Y}^{t}$ are independent in $t$, this expression defines a Markov process on $R_{\infty}^{+}$. Moreover, since the deterministic operator $\mathbf{D}(\cdot)$ as well as the distribution of $\mathbf{Y}^{t}$ do not depend on time, the process is homogeneous in time.

Conceptually, this phase space is formed by the value characterizing the highest productivity which is potentially attainable at any time in the industry (the first coordinate), capitals per unit of output (the first $k$ coordinates in each cohort, that is, a $2 k$ box in the above structure) and individual capital stocks (the last $k$ coordinates in each cohort: that is, to a capital ratio placed at the $j$-th position corresponds the capital placed at the $(k+j)$-th position) of all firms that stay alive. Therefore, if $q_{k+i}^{n}(t)>0$ for some $i=1,2, \ldots, k$ and $n \leq t$, then a firm with $q_{i}^{n}(t)$ as capital per unit of output came to the industry at $t-n$, has been alive until $t$, that is, has manufactured $n-1$ times, and continues to produce during the $t$-th time 
period. The representation via a direct sum seems to be a handy way of explicitly capturing the dynamic of cohorts.

The formulas for $\mathbf{D}^{i}(\cdot), i \geq 2$, reflect our investment rule together with the assumption that the capital ratio remains constant through the life time of a firm. In analogy with Eq. (2.1), they are capturing the dynamic of capital stocks (but more precisely Eq. (2.1) refers to productive capacities). The indicators are needed because of the death rule ${ }^{5}$. The relation $\mathscr{A}_{j}^{i}(\mathbf{q})$ gives the criterion that a firm from the $i$-th box placed at the $j$-th position continues to manufacture given the state of the industry q. As from above, the first coordinate carries the value determining the highest productivity attainable in the industry. The further $2 k$ block is zero to host newcoming firms. The next $k$ ones reflect the learning rule on improvement of productivity adopted by newcoming firms. Finally, the last $k$ coordinates of $\mathbf{D}^{1}(\cdot)$ are defined according to our investment rule.

Given this formal description of this process of industry evolution, let us proceed to the analysis of its long run behavior.

\subsection{Asymptotic properties of the industry}

Define $\mathscr{B}^{\infty}$ the minimal $\sigma$-field in $R_{\infty}$ generated by sets of the following form

$$
\mathscr{A}=A \otimes\left[\bigotimes_{j=1}^{\infty} A^{j}\right],
$$

where $A$ designates a set from the $\sigma$-field of Borel sets $\mathscr{B}$ on the real line, and $A^{j}$ being a set from the $\sigma$-field of Borel sets $\mathscr{B}_{j}$ in $R_{j}$. For every such set $\mathscr{A}$ one step transition probability of process Eq. (2.3) reads

$$
p^{1}(\mathbf{q}, \mathscr{A})=P\{\mathbf{D}(\mathbf{q})+\mathbf{Y} \in \mathscr{A}\}=P\left\{\mathbf{Y}^{*} \in A \otimes A_{1}\right\} \chi_{\mathbf{D}(\mathbf{q}) \in \underset{i=2}{\otimes} A^{i}}
$$

Here $\mathrm{Y}^{*}$ stands for the $(2 k+1)$-dimensional vector whose coordinates coincide with first $2 k+1$ coordinates of a generic vector $\mathbf{Y}$ having the same distribution as $\mathbf{Y}^{t}$, $t \geq 0$.

To study the ergodic properties of process (2.3), we need the following condition which is due to Doeblin (see Doob, 1953, p. 192).

There is a finite positive measure $\phi(\cdot)$ with $\phi\left(R_{\infty}^{+}\right)>0$ and a positive number $\delta$ such that for all $\mathbf{q} \in R_{\propto}^{+} p^{1}(\mathbf{q}, \mathscr{A}) \leq 1-\delta$ if $\phi(\mathscr{A}) \leq \delta$.

For a set $\mathscr{A}$ as in (2.4) let $\phi(\mathscr{A})=P\left\{\mathbf{Y}^{*} \in A \otimes A_{1}\right\}$. From (2.4) it follow that $p^{1}(\mathbf{q}$, $\mathscr{A}) \leq \phi(\mathscr{A})$. Since $\phi\left(R_{\infty}^{+}\right)=P\left\{\mathrm{Y}^{*} \in[0, \infty) \otimes \mathrm{R}_{1}^{+}\right\}=1$, restricting ourselves to $\delta \leq 1 /$ 2 , we get that, if $\phi(\mathscr{A}) \leq \delta$ then $p^{1}(\mathbf{q}, \mathscr{A}) \leq \delta \leq 1-\delta$. Thus, Doeblin's condition holds for this choice of $\phi(\cdot)$ and all $\delta \in(0,1 / 2]$.

Now, by Theorem 5.7 from Doob (1953) (p. 214), we see that

\footnotetext{
${ }^{5}$ In particular, applied to the first $k$ coordinates in a cohort, they prevent from carrying over the capital ratios of firms that have died. However, the use of the death indicators to the first $k$ coordinates is basically a matter of taste: without relevance for the conclusions, dropping them implies that the capital ratios of dead firms are in the structure of the model forever.
} 


$$
\pi(\mathbf{q}, \mathscr{A})=\lim _{n \rightarrow \infty} \frac{1}{n} \sum_{t=1}^{n} p^{t}(\mathbf{q}, \mathscr{A})
$$

defines for each $\mathbf{q} \in R_{\infty}^{+}$a stationary absolute distribution. Here $p^{t}(x, \cdot)$ stands for the transition probability in $t$ steps, that is,

$$
p^{t}(\mathbf{q}, \mathscr{A})=\int_{R_{+\infty}^{+}} p^{t-1}(\mathbf{y}, \mathscr{A}) \mathrm{d} p^{1}(\mathbf{q}, \mathbf{y}), \quad t \geq 2 .
$$

The stationary distribution $\pi(\mathbf{q}, \cdot)$ turns out to be the same, that is $\pi_{\mathfrak{f}}(\cdot)$ for all $\mathbf{q}$ belonging to the same ergodic set $£$ (see Doob, 1953, p. 210). It has the following generic property

$$
\int_{\mathfrak{f}} p^{1}(\mathbf{x}, \mathscr{A}) \mathrm{d} \pi_{\mathfrak{f}}(\mathbf{x})=\pi_{\mathfrak{f}}(\mathscr{A}) .
$$

In general, it is not possible to find an explicit expression for $\pi_{\mathfrak{f}}(\cdot)$ from this relation.

Thus, we may only obtain the following result concerning ergodicity of process Eq. (2.3).

Theorem 2.1. For every set Al given by Eq. (2.4) with probability one

$$
\frac{1}{n} \sum_{t=1}^{n} p^{t}\left(\mathbf{Y}^{0}, \mathscr{A}\right) \rightarrow \pi\left(\mathbf{Y}^{0}, \mathscr{A}\right)
$$

as $n \rightarrow \infty$. Here $\pi\left(Y^{0}, \cdot\right)$ is a stochastic probability measure (since it depends on $Y^{0}$ ), with $\pi_{f}(\cdot)$ for any elementary outcome $\omega \in \Omega$ whereby $Y^{0}$ belongs for this elementary outcome to an ergodic set $£$.

Consider the implications of this result in terms of path dependency. On the one hand, Doeblin's condition implies that events occuring at $t$ and $t+n$ are getting more and more statistically independent as $n$ increases. Thus, the impact of the initial state vanishes as time goes on. Should one be able to prove that there is a single ergodic set, then the limit of time averages in Eq. (2.6) would not depend on the initial state, and hence the lack of path-dependency. On the other hand, the limit in Eq. (2.6), in general, does depend on the initial state. But the dependency acts in a way that such limit turns out to be the same for all initial states belonging to the same ergodic set. Therefore, there might indeed be some path-dependency which is governed by a partition of $\Omega$. Note also that this partition, in general, turns out to be less fine than the one given by $\mathbf{Y}^{0}$.

Theorem 2.1 implies that, for every uniformly bounded characteristic of the industry, its time averages converge with probability one to a limit which is a deterministic function of the initial state in the sense given above. Unfortunately, unlike for the model considered by Winter et al. (1997), some of the most important dynamic characteristics such as, for example, the total productive capacity, here are not uniformly bounded. Hence, Theorem 2.1 does not allow for immediate conceptual conclusions analogous to the ergodicity result presented in the foregoing work. On the other hand, one is still able to establish convergence of time averages of other aggregate variables such as the gross profit rate. Set 


$$
r_{t}=\frac{Q_{t} H\left(Q_{t}\right)}{\sum_{i=1}^{\infty} \sum_{j=1}^{k} q_{k+j}^{i}(t)}
$$

for the gross profit rate at $t \geq 1$. Since there is no production at $t=0, r_{0}=0$. As a consequence of Theorem 2.1 we have the following statement.

Corrollary 2.1. If $x H(x) \leq$ const for $x \rightarrow \infty$, then with probability one

$$
\frac{1}{n} \sum_{i=1}^{n} r_{i} \rightarrow \int_{R_{x}^{+}} \frac{Q(\mathbf{y}) H(Q(\mathbf{y}))}{\sum_{i=1}^{\infty} \sum_{j=1}^{k} y_{k+j}^{i}} \mathrm{~d} \pi\left(\mathbf{Y}^{0}, \mathrm{~d} \mathbf{y}\right)
$$

as $n \rightarrow \infty$. Here for a vector $\mathbf{y}$ of the form Eq. (2.2)

$$
Q(\mathbf{y})=\sum_{s=1}^{k}\left[y_{k+s}^{1} \exp \{y\} / y_{s}^{1}+\sum_{i=2}^{\infty} y_{k+s}^{i} / y_{s}^{i}\right] .
$$

Indeed, $Q_{t} H\left(Q_{t}\right) \leq$ const by hypothesis. Also

$$
\sum_{i=1}^{\infty} \sum_{s=1}^{k} q_{k+j}^{i}(t) \geq \sum_{i=1}^{k} \theta^{(t-1) k+i} \geq k c, \quad t \geq 1 .
$$

Hence $r_{t} \leq$ const $/ k c<\infty$. Which implies that Eq. (2.7) follows from Eq. (2.6).

Note that if $\lim _{x \rightarrow \infty} x H(x)=H$, then

$$
\frac{1}{n} \sum_{i=1}^{n} r_{i} \rightarrow H \int_{R_{x}^{+}} \frac{\mathrm{d} \pi\left(\mathbf{Y}^{0}, \mathbf{y}\right)}{\sum_{i=1}^{\infty} \sum_{j=1}^{k} y_{k+j}^{i}} .
$$

As mentioned, the total productive capacity of this industry unboundedly increases as time goes on. More precisely, we have the following statement.

Lemma 2.1. The total productive capacity $Q_{t}$ of the industry goes to infinity with probability one as $t \rightarrow \infty$.

The lemma is proved in the Appendix.

Now let us study the mortality of firms in this competitive environment.

Theorem 2.2. If $\varepsilon>0$, then every firm dies in a finite random time with probability one.

The proof is given in the Appendix. The argument exploits the fact that the total productive capacity of the industry grows without bound, essentially due to the increasing capital efficiency embodied - in probability - in the new capital vintages, which implies that every firm with a fixed capital per unit of output starts shrinking from a finite random time with probability one. 
Having shown the unbounded increase of productive capacity as time goes on, let us now characterize its rate of growth.

Theorem 2.3. With probability one $\exp \{-\alpha t\} Q_{t} \rightarrow \infty$ as $t \rightarrow \infty$ for every $\alpha<E \xi$. Moreover, if

$$
\lim _{x \rightarrow \infty} H(x) x=0
$$

then with probability one $\exp \{-\alpha t\} Q_{t} \rightarrow 0$ as $t \rightarrow \infty$ for every $\alpha>E \xi$.

The theorem is proved in the Appendix.

Remark 2.1. The same result obtains if, instead of Eq. (2.8), we require that

$$
\limsup _{x \rightarrow \infty} H(x) x<d \frac{k v c a}{\lambda b} .
$$

Now, if for a positive number $H$ a demand function decreases as $H / x$ for $x \rightarrow \infty$, then, keeping all other parameters of the model involved in the right hand side of Eq. (2.9) fixed, one can ensure Eq. (2.9) just increasing $k$. Thus, for such demand functions the second statement of Theorem 2.3 always holds true if the number of newcoming firms is large enough.

We have showed that the productive capacity of the industry always grows faster than $\exp \{t \alpha\}$ for every $\alpha<E \xi$. If, additionally, the demand function declines fast enough (see Eq. (2.8) or Eq. (2.9)), then the productive capacity always grows slower than $\exp \{t \alpha\}$ for every or $\alpha>E \xi$. Consequently, the threshold value $E \xi$ is the only candidate for the growth rate in the class of exponential functions of time. This growth is entirely due to the increasing efficiency of newcoming firms, and is not dependent upon the investment mechanism employed in the model. Interestingly, one is not able to prove that $\exp \{-t E \xi\} Q_{t}$ converges to a limit as $t$ increases. Indeed, here we are facing with a variety of growth regimes. Each of them is determined probabilistically by the development path (i.e. also a particular 'technological trajectory') and is deviating from the main trend, $\exp \{t E \xi\}$, by a value vanishing as $t \rightarrow \infty$ faster than $\exp \{-t \beta\}$ for every $\beta>0$. Hence, these deviations are not detectable if we restrict ourselves to the class of exponential functions of time. To understand why this happens, let us consider the asymptotic behavior of the value $V_{t}$ giving the lower bound for the total productive capacity since $Q_{t+1} \geq V_{t}$.

The random variable $V_{t}$ is a product of the two other ones: $\exp \left\{A_{t}\right\}$ and $\Theta^{t}$. The latter, $\Theta^{t}$ does not contribute to the growth rate since its distribution does not depend on $t$, being a convolution of $k$ copies of $\theta / \zeta$. Hence, let us focus on $A_{t}$. We have that

$$
A_{t}=\sum_{i=0}^{t} \xi^{i}=(t+1) E \xi+\sum_{i=0}^{t} \xi_{*}^{i}
$$

where $\xi_{*}^{i}=\xi^{i}-E \xi^{i}, i \geq 0$. The law of iterated logarithm (see Loève, 1955, p. 260) implies that 


$$
P\left\{\limsup _{t \rightarrow \infty} \frac{\left|\sum_{i=0}^{t} \xi_{*}^{i}\right|}{\sqrt{2 D \xi(t+1) \ln \ln D \xi(t+1)}}=1\right\}=1,
$$

taking into account that the random variables $\xi$ and $\xi-E \xi$ have the same variance. Consequently, there are subsequencies $t_{n}^{+}, n \geq 1$, and $t_{n}^{-}, n \geq 1$, such that with probability one

$$
\lim _{n \rightarrow \infty} \frac{\sum_{i=0}^{t_{n}^{+}} \xi_{*}^{i}}{\sqrt{2 D \xi\left(t_{n}^{+}+1\right) \ln \ln D \xi\left(t_{n}^{+}+1\right)}}=1
$$

and

$$
\lim _{n \rightarrow \infty} \frac{\sum_{i=0}^{t_{n}^{-}} \xi_{*}^{i}}{\sqrt{2 D \xi\left(t_{n}^{-}+1\right) \ln \ln D \xi\left(t_{n}^{-}+1\right)}}=1 .
$$

Consequently, as $n \rightarrow \infty$

$$
\exp \left\{A_{t_{n}^{+}}-\left(t_{n}^{+}+1\right) E \xi\right\} \sim \exp \left\{\sqrt{2 D \xi\left(t_{n}^{+}+1\right) \ln \ln D \xi\left(t_{n}^{+}+1\right)}\right\}
$$

and

$$
\exp \left\{A_{t_{n}^{-}}-\left(t_{n}^{-}+1\right) E \xi\right\} \sim \exp \left\{-\sqrt{2 D \xi\left(t_{n}^{-}+1\right) \ln \ln D \xi\left(t_{n}^{-}+1\right)}\right\}
$$

with probability one. Thus, what remains in $\exp \left\{A_{t}\right\}$ if we remove its main part, $\exp \{(t+1) E \xi\}$, can be converging (along certain sequencies) with probability one to both infinity and zero. Hence, the remaining value does not have any definite rate of growth as time goes on. Also, Eq. (2.10) shows that there is no hope to find a finite limit for $\exp \{-t E \xi\} Q_{t}$ as $t \rightarrow \infty$. Indeed, since $Q_{t+1} \geq V_{t}$, by Eq. (2.10) we get that with probability one

$$
\exp \left\{-t_{n}^{+} E \xi\right\} Q_{t_{n}^{+}} \geq \exp \left\{-t_{n}^{+} E \xi\right\} V_{t_{n}^{+}-1} \rightarrow \infty \quad \text { as } \quad n \rightarrow \infty .
$$

Hence, we find here a path-dependency property of the model. While we have proved that the threshold value of the rate of growth is exponential, it is history which selects the exact value of such rate.

Remark 2.2. With the foregoing setting one may easily endogenize the entry rate by making it stochastically dependent on some system variable, e.g. current profitability, without qualitatively affecting the results. Let $k$ be the maximum number of entrants. Fix positive numbers $p_{0}, p_{1}, \ldots, p_{k}$,

$$
\sum_{i=0}^{k} p_{i}=1 .
$$

Let $\Phi(\cdot)$ be a decreasing function mapping $[0, \infty)$ to $[0,1]$. For example, $\Phi(x)=$ $\exp (-\Phi x), \Phi>0$. The random variable $\gamma^{t}\left(Q_{t}\right)$ governing the number of firms that enter the industry at $\mathrm{t}$ can be as follows 


$$
\gamma^{t}(x)=\left\{\begin{array}{l}
0, \quad \text { with probability } p_{0} \Phi(H(x)), \\
s, \quad \text { with } \text { probability } \frac{p_{s}}{1-p_{0}}\left[1-p_{0} \Phi(H(x))\right],
\end{array}\right.
$$

where $1 \leq s \leq k$. For any deterministic $x_{t}$, the random variables $\gamma^{t}\left(x_{t}\right)$ are assumed to be stochastically independent in $t$. They also do not depend upon $\xi$, $\zeta$ and $\theta$.

Remark 2.3. Also death rules can be endogenized in this basic framework. For example, one could make them dependent on the total productive capacity of the industry at each $t$ : that is, a firm is dead and does not participate in the industry evolution thereafter if its productive capacity is less than $\varepsilon Q_{t}$, where $\varepsilon \in(0,1)$ denotes some critical threshold value. Somewhat related, as we show in Winter et al. (1997), the model withholds also extensions whereby the investment rates depend upon some threshold profit margins.

\subsection{Different time-scales of technological learning}

So far one has assumed that production, entry and learning (by entrants) all take place on the same time-scale (i.e. at each 'period'). However, the model can be extended to account also for a timing of innovative 'events' asynchronous vis-à-vis production and entry. Suppose, for example, that the enlargement of innovative opportunities occurs at a slower pace.

This phenomenon may be formalized in the following way.

Let $T_{n}, n \geq 0$, be an increasing sequence of positive integers such that $T_{0}=0$ and $T_{n+1}-T_{n} \geq 1$. Also, let

$$
A_{n+1}=A_{n}+\xi^{n+1}, \quad n \geq 0,
$$

and the levels of capital per unit of output of all firms to be coming during the time interval $\left(T_{n}, T_{n+1}\right)$ from the distribution concentrated on $\left[\exp \left\{-A_{n}\right\} a, \exp \{-\right.$ $\left.\left.A_{n}\right\} b\right]$. So, capital ratios of the $k$-firms coming at time $t$ are determined as $\exp \left\{-A_{n}\right\} \zeta^{i}, t k+1 \leq i \leq(t+1) k$ provided that $T_{n} \leq t<T_{n+1}$. Here $\xi^{n}, n \geq 0$, and $\zeta^{i}, i \geq 1$, are mutually independent collections of realizations of $\xi$ and $\zeta$.

The sequence $T_{n}, n \geq 0$, characterizes the slower pace of generation of potential innovations as compared to the timing of manufacturing 'periods'. Hence the main component of the rate of growth of capital productivity for individual entrants and for the whole industry (under some additional assumptions, cf. Theorem 2.3 and Remark 2.1) as $t \rightarrow \infty$ is determined by the function $\exp \left\{T^{-1}(t) \cdot E \xi\right\}$. Here $T^{-1}(\cdot)$ designates an inverse function to $T(\cdot): n \rightarrow T_{n}$. For example, if $T_{n}=s \cdot n$ for an integer $s>1$, then

$$
\lim _{t \rightarrow \infty} \frac{\exp \left\{T^{-1}(t) \cdot E \xi\right\}}{\exp \left\{\frac{E \xi}{s} t\right\}}=1 .
$$

Similarly, if $T_{n}$ equals to the integer part of $\exp \{\alpha \cdot n\}$ for a real $\alpha>0$ (and for sufficiently large $n$ ), then 


$$
\lim _{t \rightarrow \infty} t^{-E \xi / \alpha} \exp \left\{T^{-1}(t) \cdot E \xi\right\}=1 .
$$

Clearly, asynchronous (and slower) paces of expansion of innovative opportunities will imply also slower rates of growth of output of the industry under consideration.

\subsection{A computer simulation}

To illustrate some quantitative properties of the model, let us consider a computer simulation ${ }^{6}$. The run presented here has the following parametrization: $k=12, v=1, d=0.3, \lambda=0.6, a=2, b=6, c=0.02, h=0.04, \varepsilon=0.5$. The demand function is $H(x)=4.1667 \exp (-0.1 x)$. The random variable $\zeta$ is uniformly distributed over $[a, b], \xi$ is uniformly distributed over $[0,0.01]$, and the capitals of newcoming firms are uniformly distributed over $[c, h]$.

Figs. 1 and 2 present the dynamic of prices. While prices decline to 0 with persistent fluctuations, the total productive capacity grows over time with qualitatively similar fluctuating patterns, whereby the amplitude of fluctuations themselves does not dampen out over time (in fact, in absolute terms, they increase): see Figs. 3 and 4 .

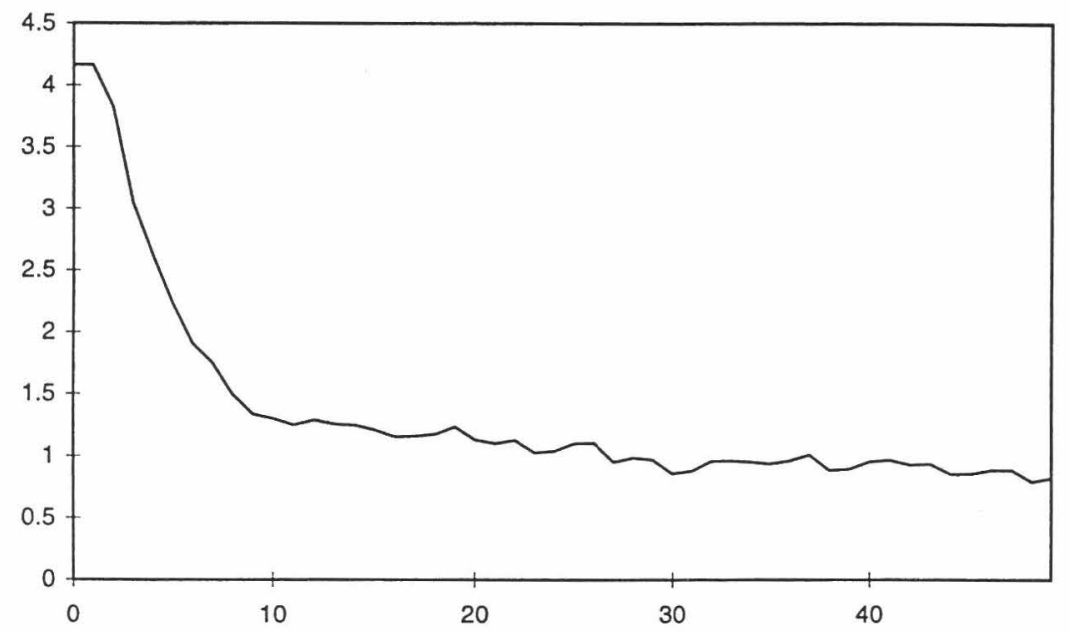

Fig. 1. Price $H\left(Q_{t}\right)$ for $1 \leq t \leq 50$.

\footnotetext{
${ }^{6} \mathrm{~A}$ lot of simulations of this kind has been undertaken based on a program from the laboratory for simulation development (LSD), a package providing an environment for implementation of simulation models developed at the International Institute for Applied Systems Analysis (IIASA). It is publicly available via Internet (see also Valente, 1997). We have tried several runs with the same parametrization (since each 'history' is a particular sample path of the stochastic process defined by the above model) and we also experimented with different parametrizations. Even if we did not perform any more rigorous sensitivity analysis, the qualitative feature of the example which follows hold throughout all the performed experiments.
} 


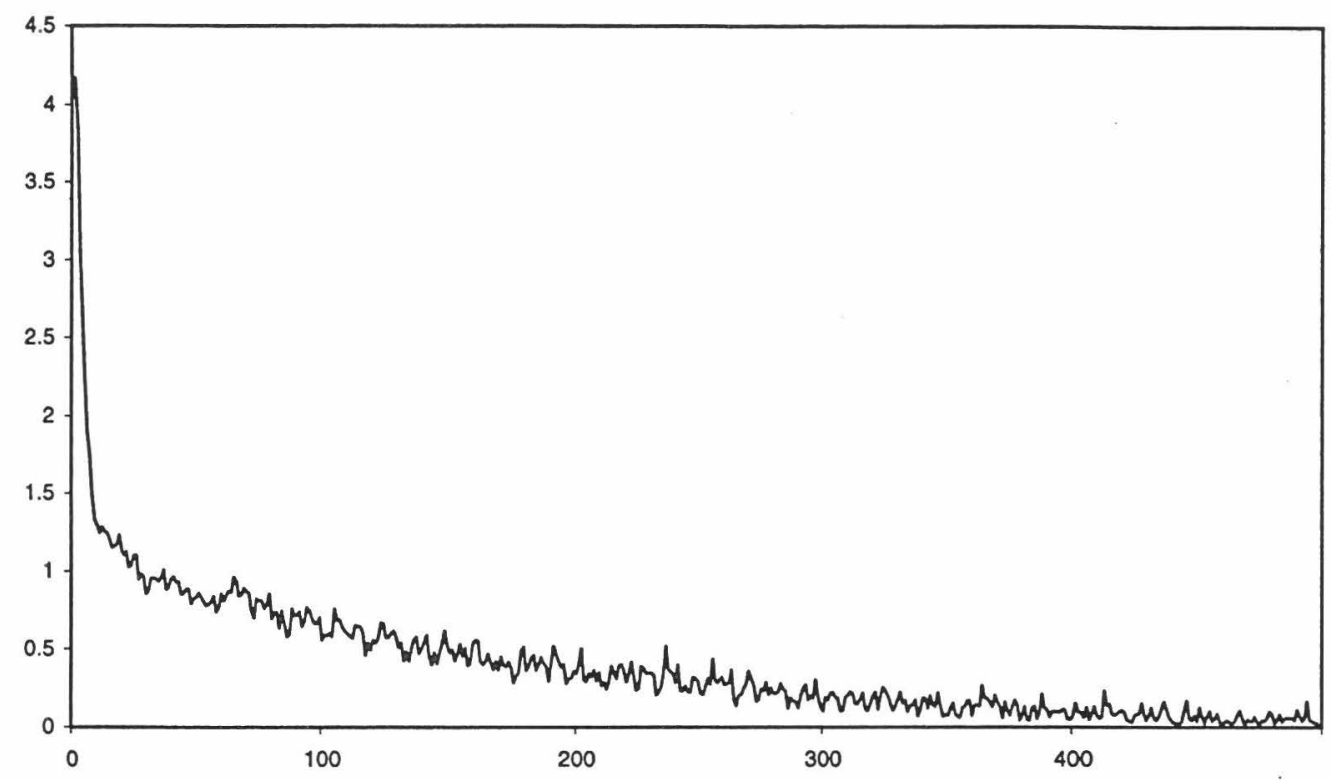

Fig. 2. Price $H\left(Q_{t}\right)$ for $1 \leq t \leq 500$.

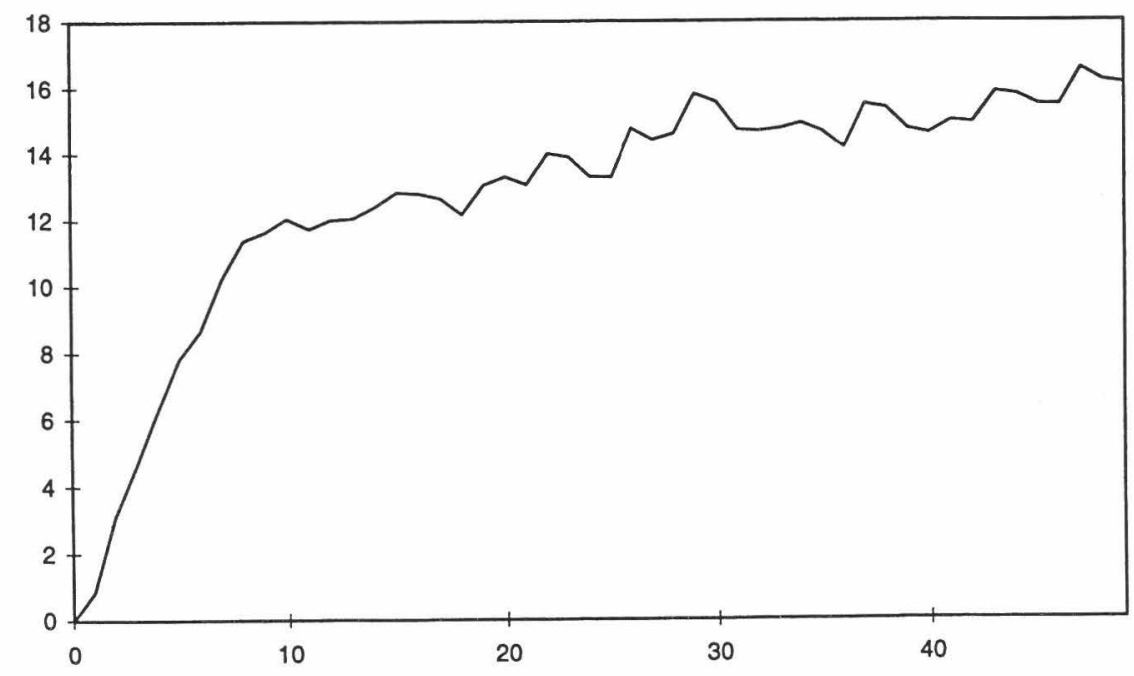

Fig. 3. Productive capacity $1 \leq t \leq 50$.

Fig. 5 illustrates the dynamic of the number of firms in the industry. After an initial period of growth it declines, and then fluctuates around a fixed level. As already noted in Winter et al. (1997), phenomena looking like 'shakeouts' at some point in the early history of an industry appear to be a rather generic property plausibly associated with a changing selection regime. At the start, the 'carrying capacity' of the market exceeds the effective supply. So, in a sense, there is 'room 
for everyone'. At some point, as total supply increases, competitive conditions become more stringent and market selection rather quickly starts affecting growth and survival of lower-efficiency firms'. This change in 'market selection regime' is illustrated also by the dynamics of the concentration measures of the industry (see

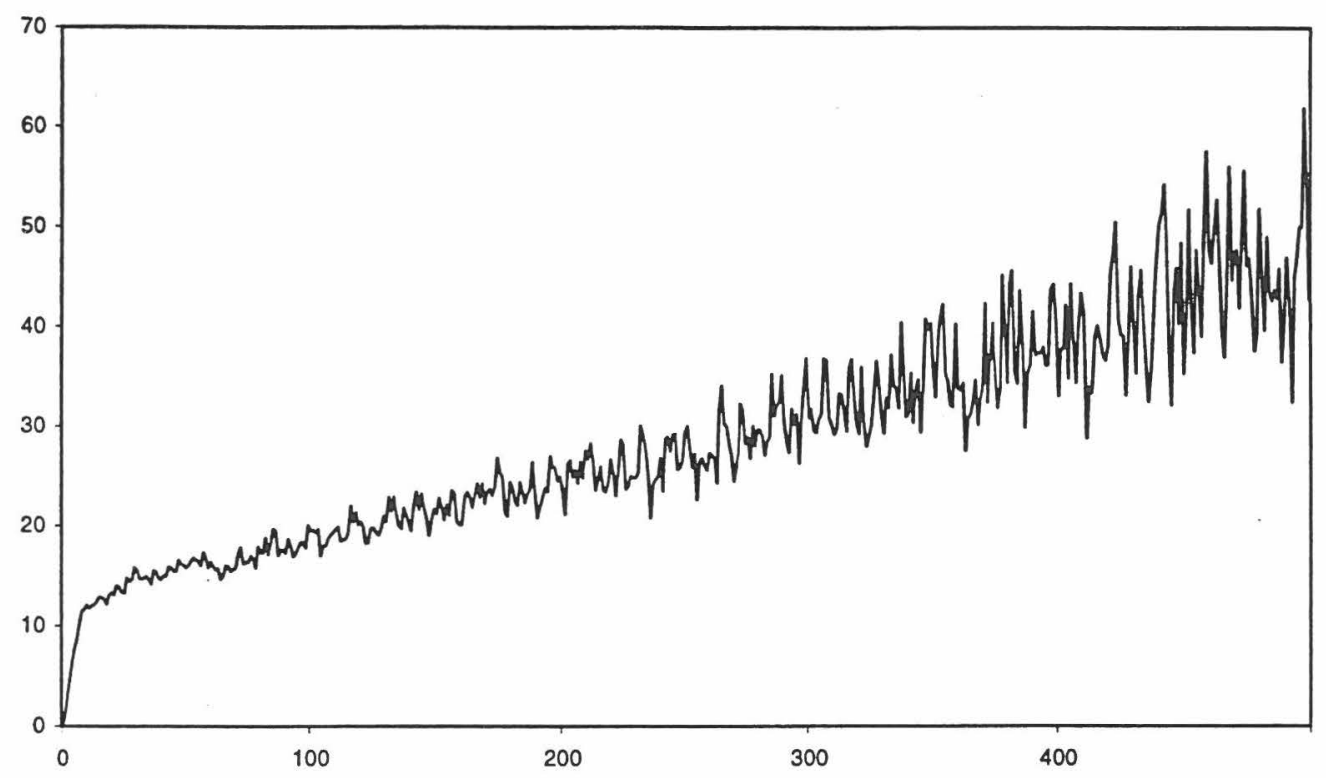

Fig. 4. Productive capacity for $1 \leq t \leq 500$.

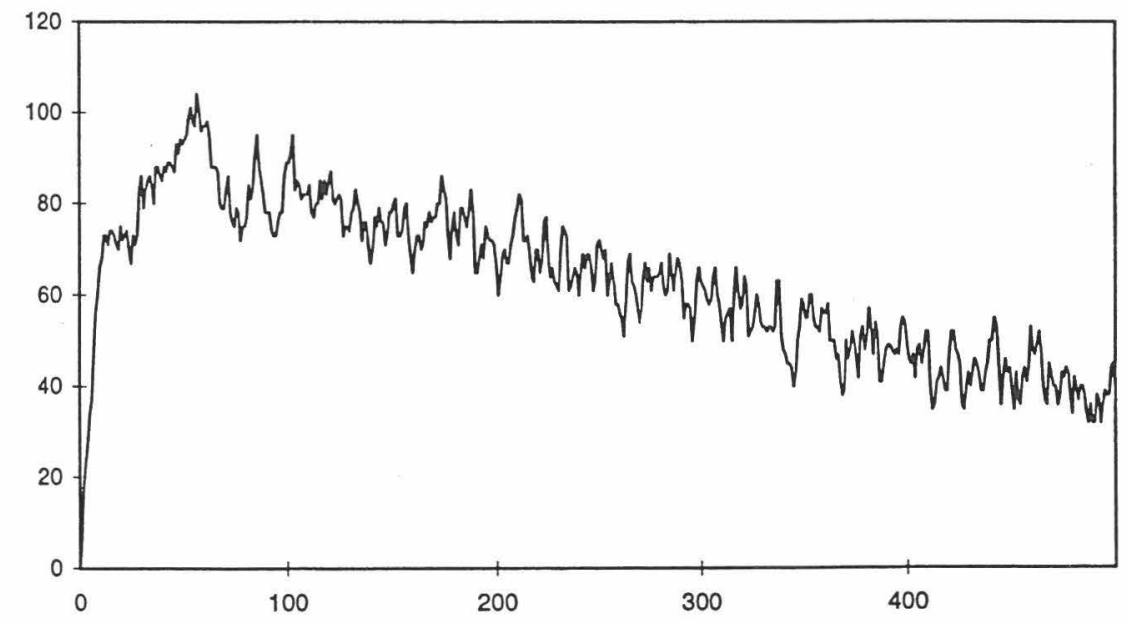

Fig. 5. Total number of firms $0 \leq t \leq 500$.

\footnotetext{
${ }^{7}$ In many respects, the phenomenon recalls the 'density dependent selection' emphasized in 'organizational ecology' models (cf. among others Hannan and Freeman, 1989 and Carroll, 1997).
} 


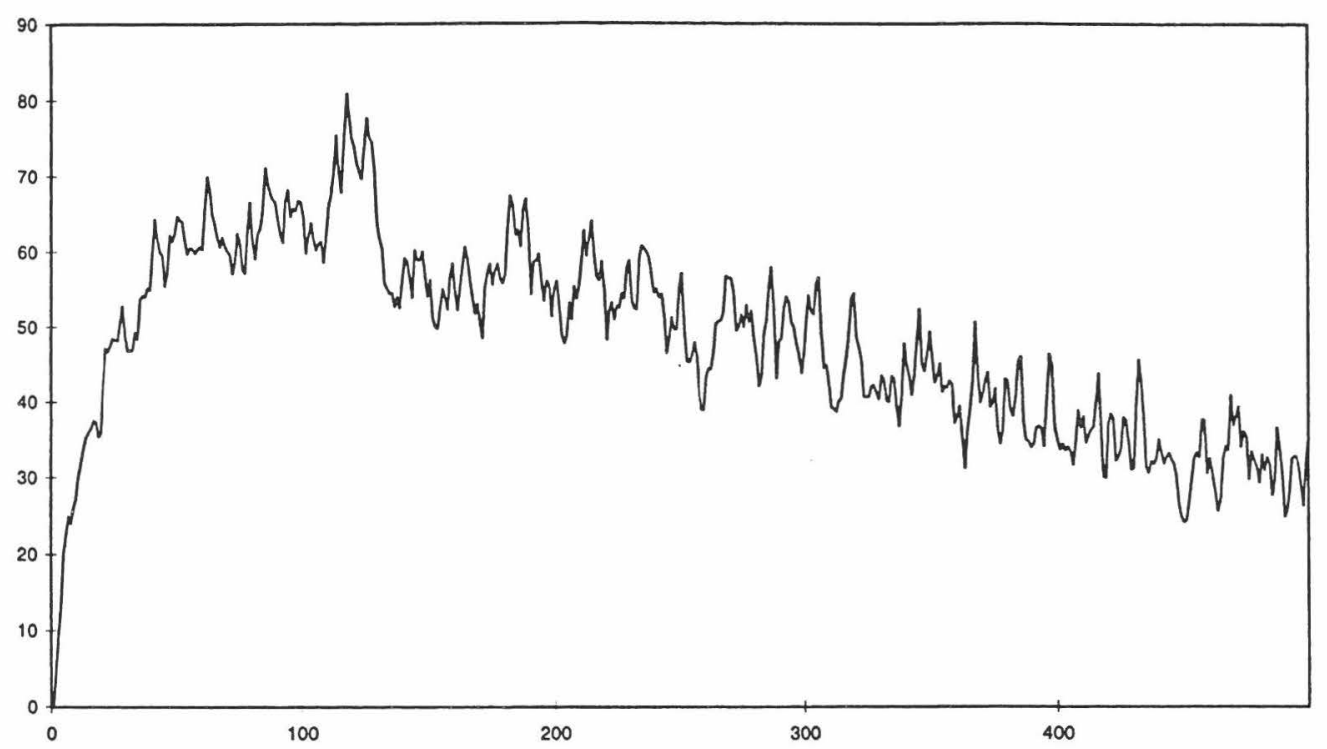

Fig. 6. Equivalent number of firms from the Hirfindhal index $1 \leq t \leq 500$.

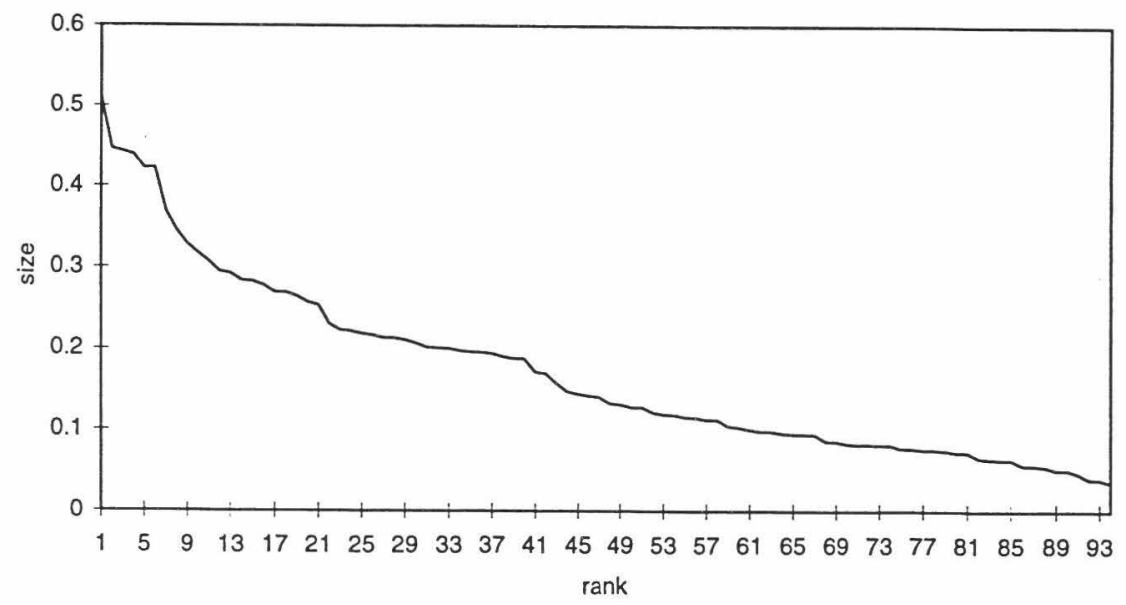

Fig. 7. Size distribution for $t=50$.

Fig. 6 for the 'equivalent number' associated with the Hirfindhal index of concentration $^{8}$ : concentration falls (i.e. the equivalent number increases) up to the 'shake out' phase and then increases thereafter. Figs. 7 and 8 provide two

${ }^{8}$ Calling $s_{i}(t)$ the market share of the $i$-th firm at $t$, the concentration index is

$$
H(t)=\sum_{i=1}^{n_{t}} s_{i}(t)^{2}
$$

The 'equivalent number' $1 / H(t)$ corresponds to the number of firms of equal size which would yield the same value of $H(t)$. 
snapshots, measured in terms of productive capacity for $t=50$ and $t=500$, where firms are ranked according to their size. What is observed here is something rather close to the Pareto law (see, for example, Ijiri and Simon, 1974) ${ }^{9}$. Fig. 9 provides the life time distribution of firms for $1 \leq t \leq 500$ which died before $t=500$. Life time here means the number of production cycles the firm performs before it dies.

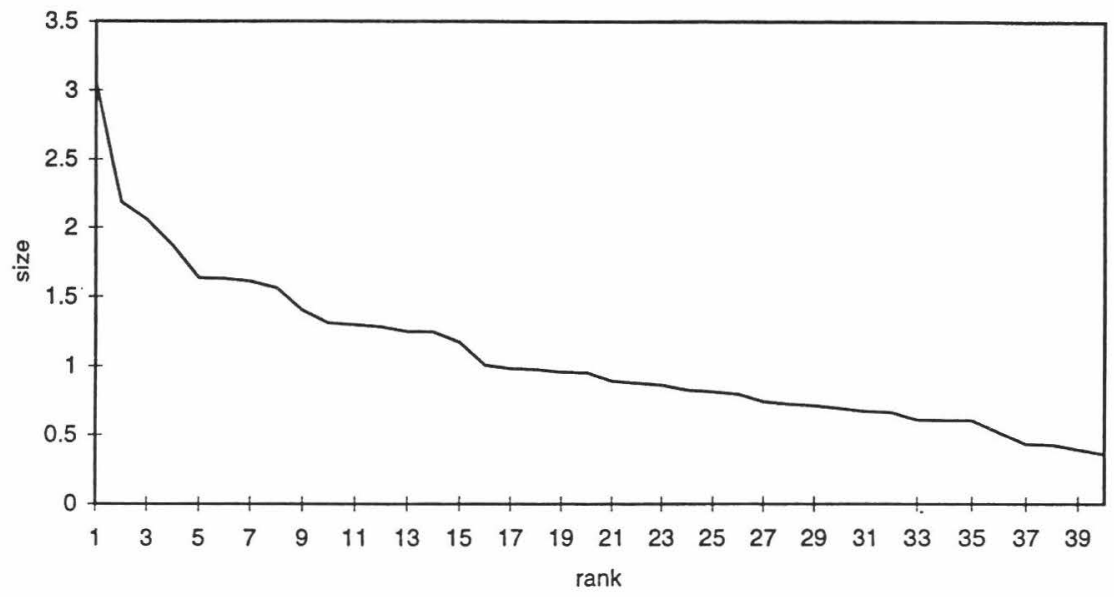

Fig. 8. Size distribution for $t=500$.

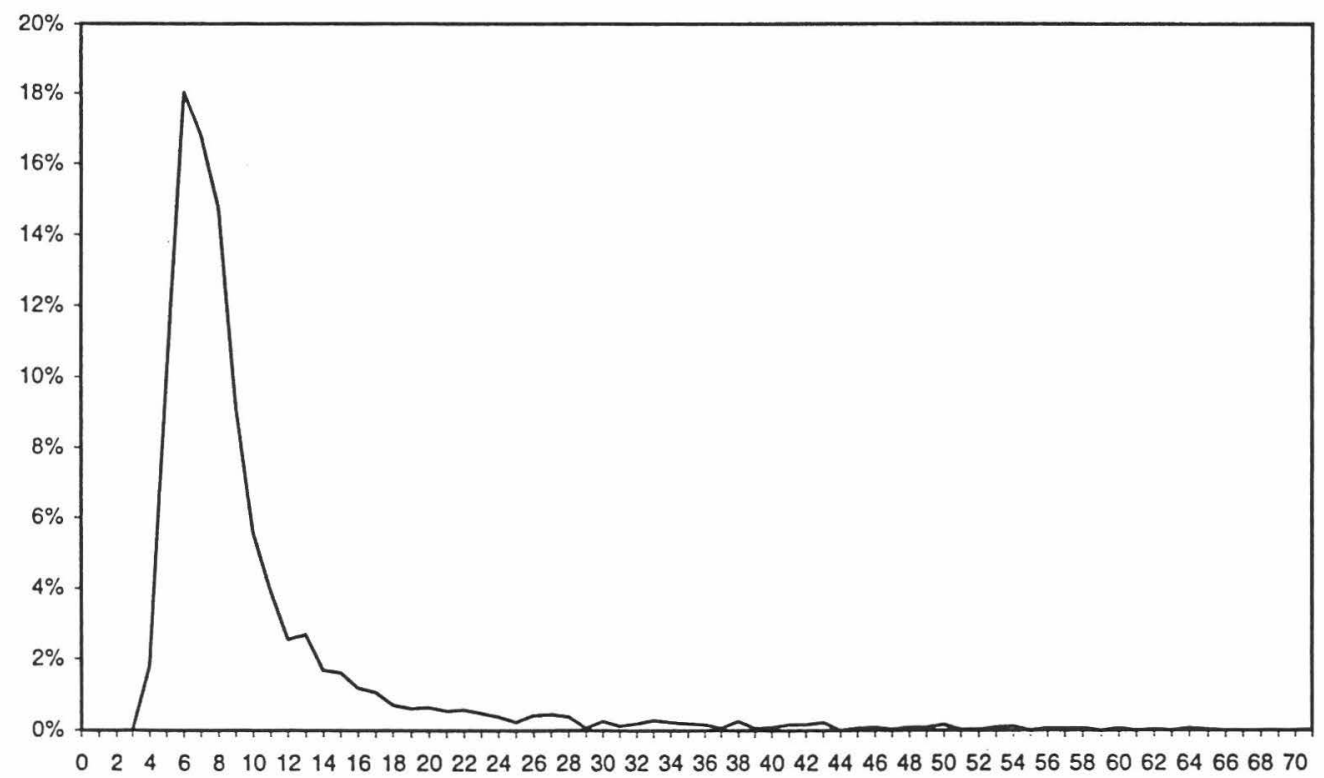

Fig. 9. Life time distribution of firms for $1 \leq t \leq 500$.

\footnotetext{
${ }^{9}$ Namely, in one of its versions, for a sample of firms ranked according to their size, the size $s$ and the rank $r$ of a firm are related in the following manner: $s r^{\beta}=A$, where $\beta$ and $A$ are positive constants.
} 


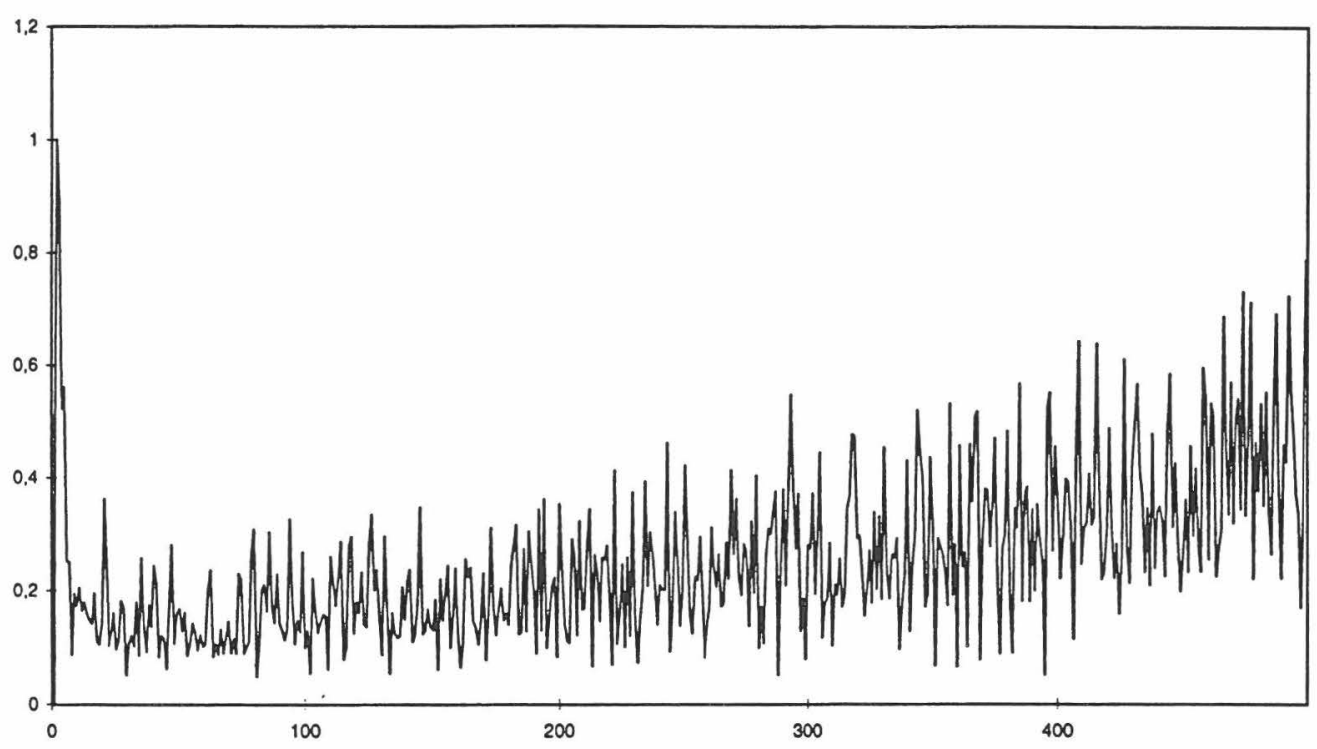

Fig. 10. Turbulence index for $1 \leq t \leq 499$.

(So for example, in Fig. 9, around 18\% of all firms which were born and died before $t=500 \mathrm{did}$ die when they were 6-periods old, etc.) Again, the mortality patterns appear to be quite in tune with the evidence, with high mortality rates shortly after birth and a (relative thin) tail of firms with much higher longevity (more on this type of evidence in Hannan and Freeman, 1989; Baldwin, 1995; Geroski, 1995; Carroll, 1997). As known, this survival patterns are sometimes interpreted especially in the 'organizational ecology' perspective - as the outcome of the differential adaptation of subsets of firms in the population. Notwithstanding the likely importance of the latter phenomena, our results here seem to suggest that a distribution of mortality rates which peaks in the early infancy, with a long but thin tale of old survivors might be a rather generic property of a large class of evolutionary processes characterized by heterogenous entry and market selection (cf. also the simulation results in Winter et al., 1997) ${ }^{10}$.

Finally, Fig. 10 vividly illustrates the evolutionary proposition that whatever persistent regularity emerges in the aggregate, that is likely to be the collective outcome of an ever-lasting microeconomic turbulence. Define a 'turbulence index'

$$
T(t)=\sum_{i=1}^{n_{t}}\left|s_{i}(t)-s_{i}(t+1)\right|+\sum_{j=1}^{k} \exp \left\{A_{t}\right\} \theta^{t k+j} / \zeta^{t k+j} .
$$

that is, the sum of the absolute values in the changes of market shares from one period to the next (including gross entry at $t$ ). As Fig. 10 shows, market turbulence persists - and, if anything tends to increase, throughout the history of the industry.

\footnotetext{
${ }^{10}$ A fortiori, one should expect this property to apply also to those circumstances wherein also incumbents are allowed to learn, as in a forthcoming model by the authors, currently in progress.
} 


\section{An alternative dynamic setting: increasing productivity of labor}

\subsection{Main assumptions}

Now we turn to symmetric opposite assumptions compared to the model above and assume that learning concerns only labor productivity ${ }^{11}$. As above, we have an industry evolving in discrete time $t=0,1, \ldots$. At $t=0$ there are no firms ready to manufacture, but $k$ firms come to the industry. They will start producing at $t=1$. At time $t \geq 1$ the industry consists of $n_{t}$ firms which are involved in manufacturing and new firms that enter at $t$ and will participate in the production process from $t+1$ on. As in the earlier version of the model we have uniformly for the whole industry:

$v$ - price per unit of physical capital, $v>0$,

$d$ - depreciation rate, $0<d \leq 1$,

$C$ - capital per unit of output, $C>0$.

Here, however, the competitiveness of any firm in the industry is determined by its variable costs per unit of output. Let us designate it by $m_{i}$ for the $i$-th firm. In this competitive environment only newcomers learn how to improve (in probability) the productivity of labor. As time goes on the lowest variable costs present in the industry decreases. In particular, we have the following stochastic mechanism defined endogenously.

Consider a random variable $\xi$ with positive mean $E \zeta$ and a finite variance $D \xi$. Set $\zeta$ for a random variable distributed over $[a, b], 0<a<b<\infty$. For each time instant $t \geq 0$ allow for the industry to have $k \geq 1$ new firms whose variable costs are randomly determined as $\exp \left\{-A_{t}\right\} \zeta^{i} t k+1 \leq i \leq(t+1) k$. Here $A_{t+1}=A_{t}+\xi^{t+1}$, $t \geq 0, A_{0}=\xi^{0}$. Also, $\xi^{t}, t \geq 0$, and $\zeta^{i}, i \geq 1$, are mutually independent collections of realizations of $\xi$ and $\zeta$. One sees that all variable costs feasible for newcomers at time $t$ belong to $\left[\exp \left\{-A_{t}\right\} a, \exp \left\{-A_{t}\right\} b\right]$. Their distribution over this interval is governed by a realization of $\exp \left\{-A_{t}\right\} \zeta$. Thus $A_{t}$ characterizes in a probabilistic manner the highest productivity of labor attainable by newcomers in the industry at time $t$.

Alike the model above there is a decreasing continuous demand function $p=H(q)$, mapping $[0, \infty)$ in $[0, H(0)]$ such that $H(0)<\infty$ and $H(q) \rightarrow 0$ as $q \rightarrow \infty$.

Set $Q_{i}^{i}$ for the productive capacity of the $i$-th firm and $m_{i}$ for its variable costs. Then

$$
Q_{t}=\sum_{i=1}^{n_{t}} Q_{t}^{i}, \quad t \geq 1, \quad Q_{0}=0
$$

is the total productive capacity involved in manufacturing at $t$. The gross profit per unit of output at $t$ for the $i$-th firm is obviously $H\left(Q_{t}\right)-m_{i}$. Its total gross investment per unit of capital is $\lambda \max \left[H\left(Q_{t}\right)-m_{i}, 0\right] / v C$. As above, the constant $\lambda$ captures the share of the gross profit which is re-invested.

\footnotetext{
${ }^{11}$ An assumption, which, together with the constancy of capital/output ratios, seems nearer the empirical evidence.
} 
For each value of variable costs generated at $t$ we shall allow a single entrant. The initial capitals of entrants are independent realizations $\theta^{i}, i \geq 1$, of a random variable $\theta$ distributed over $[c, h], 0<c<h<\infty$. It is assumed that the realizations of $\xi, \zeta$ and $\theta$ are mutually independent random variables.

Again, as above, the death mechanism implies that a firm is dead at time $t$ and does not participate in manufacturing from $t+1$ onward if its capital at $t$ is less than $\varepsilon c, \varepsilon \in(0,1]$. The situation without mortality corresponds to the limit case when $\varepsilon=0$.

\subsection{A dynamic balance equation for industry evolution}

Consider a firm $i$ that is manufacturing at time $t$. Our investment rule implies that at the end of this production period its capital reads

$$
Q_{i}^{i} C\left\{1-d+\frac{\lambda}{v C} \max \left[H\left(Q_{t}\right)-m_{i}, 0\right]\right\} \text {. }
$$

If this value does not drop below the death threshold $\varepsilon c$, the firm continues to manufacture at $t+1$. Otherwise it dies ${ }^{12}$. Hence

$$
Q_{t+1}^{i}=Q_{t}^{i}\left\{1-d+\frac{\lambda}{v c} \max \left[H\left(Q_{t}\right)-m_{i}, 0\right]\right\} \%_{Q i}\left\{1-d+\frac{\lambda}{v C} \max \left[H\left(Q_{t}\right)-m_{i}, 0\right]\right\} \geq s c / C
$$

where $\chi_{Q i\left\{1-d+\lambda / v C \max \left[H\left(Q_{t}\right)-m_{t}, 0\right]\right\} \geq \varepsilon c / C}$ is the indicator function of the event that the firm continues to manufacture given the above death rule and the total productive capacity of the industry involved in manufacturing.

This equation describes the evolution of a single firm in business. In analogy with the formalization of the foregoing section, let us proceed to a dynamic representation of the model that reserves room for all feasible development paths of the industry.

For the space $R_{\infty}$ introduced in Section 2, define an automorphism $\mathbf{D}(\cdot)$ on $R_{\infty}$ such that

$$
\mathbf{D}(\mathbf{q})=\mathbf{D}^{1}(\mathbf{q}) \otimes\left[\bigotimes_{i=2}^{\infty} \mathbf{D}^{i}(\mathbf{q})\right],
$$

with $\mathbf{D}^{1}(\cdot): R_{\diamond} \mapsto R \otimes R_{1} \otimes R_{2}$ and $\mathbf{D}^{i}(\cdot): R_{\curvearrowright} \mapsto R_{i}, i \geq 3$. Set

$$
\begin{aligned}
& D_{1}^{1}(\mathbf{q})=q, \quad D_{s}^{1}(\mathbf{q})=0, \quad 2 \leq s \leq 2 k+1, \quad D_{2 k+j+1}^{1}(\mathbf{q})=q_{j}^{1} \exp \{-q\} \chi_{. \mathscr{o}_{j}^{\prime}(\mathbf{q})}, \\
& D_{3 k+j+1}^{1}(\mathbf{q})= \\
& \left\{q_{k+j}^{1}\left[1-d+\frac{\lambda}{v C} \max \left[H\left(\sum_{p=1}^{\infty} \sum_{s=1}^{k} q_{k+s}^{p}\right)-q_{j}^{1} \exp \{-q\}, 0\right]\right]\right\} \chi_{\alpha_{j}^{\prime}(\mathbf{q})},
\end{aligned}
$$

\footnotetext{
${ }^{12}$ In a possibly more realistic setting one could add a sort of bankruptcy rule stating that firms die, even when their size is greater than $\varepsilon c$, if their gross profits are negative (i.e. $\left[H\left(Q_{t}\right)-m_{i}\right]<0$ ). However, this modification would not qualitatively change the results that follow: rather it would simply affect death rates of 'uncompetitive' firms.
} 


$$
\begin{aligned}
& D_{j}^{1}(\mathbf{q})=q_{j}^{i} \chi_{\alpha_{j} i(\mathbf{q})}, \\
& D_{k+j}^{i}(\mathbf{q})=q_{k+j}^{i}\left\{1-d+\frac{\lambda}{v C} \max \left[H\left(\sum_{p=1}^{\infty} \sum_{s=1}^{k} q_{k+s}^{p}\right)-q_{j}^{i}, 0\right]\right\} \chi_{\alpha_{j}(\mathbf{q})},
\end{aligned}
$$

where $1 \leq j \leq k, i \geq 2, \mathscr{A}_{j}^{1}(\mathbf{q})$ designates the relation

$$
q_{k+j}^{1}\left\{1-d+\frac{\lambda}{v C} \max \left[H\left(\sum_{p=1}^{\infty} \sum_{s=1}^{k} q_{k+s}^{p}\right)-q_{j}^{1} \exp \{-q\}, 0\right]\right\} \geq \varepsilon c / C
$$

and $\mathscr{A}_{j}^{i}(\mathbf{q})$ stands for the relation

$$
q_{k+j}^{1}\left\{1-d+\frac{\lambda C}{v} \max \left[H\left(\sum_{p=1}^{\infty} \sum_{s=1}^{k} q_{k+s}^{p}\right)-q_{j}^{i}, 0\right]\right\} \geq \varepsilon c / C .
$$

We restrict ourselves to vectors $\mathbf{q}$ defined by Eq. (2.2) belonging to

$$
R_{\infty}^{+}=[0, \infty) \otimes\left[\bigotimes_{i=1}^{\infty} R_{i}^{+}\right]
$$

and set $H(\infty)=0$ for the case when the iterated sum is infinite.

The conceptual interpretation of the automorphism is very similar to the one given earlier on. The $2 k$ boxes contain data concerning cohorts, that is groups of firms which were born simultaneously. The only exception is the first box containing two cohorts and additionally (its first coordinate) the value capturing the highest productivity of labor attainable in the industry. In each cohort the first $k$ coordinates are the variable costs and the last $k$ coordinates represent productive capacities of corresponding firms. The adjustment rule for productive capacities is the same as in Eq. (3.1). (Again, the indicators prevent from carrying over the data related to dead firms.) The relation $\mathscr{A}_{j}^{i}(\mathbf{q})$ means that a firm which is placed at the $j$-th position of the $i$-th cohort continues to manufacture given the state of the industry $\mathbf{q}$.

Define infinite dimensional random vectors $\mathbf{Y}^{t}, t \geq 0$, setting

$$
\begin{aligned}
& Y_{1}^{l}=\check{\zeta}^{\prime}, \quad Y_{i+1}^{t}=\zeta^{t k+i}, \quad Y_{k+i+1}^{t}=\theta^{t k+i}, \quad i=1,2, \ldots, k, \\
& Y_{j}^{t}=0 \quad j \geq 2 k+2 .
\end{aligned}
$$

The evolution of the industry is as follows

$$
\mathbf{q}(t+1)=\mathbf{D}(\mathbf{q}(t))+\mathbf{Y}^{t+1}, \quad t \geq 0, \quad \mathbf{q}(0)=\mathbf{Y}^{0},
$$

Since $\mathrm{Y}^{t}$ are independent in $t$, this expression defines a Markov process on $R_{\infty}^{+}$. Moreover, it is homogeneous in time since the deterministic operator $\mathbf{D}(\cdot)$ as well as the distribution of $\mathbf{Y}^{t}$ do not depend on time.

This phase space is formed by the value characterizing the highest productivity which is potentially attainable so far in the industry, variable costs and productive capacities of all firms that stay alive. 


\subsection{Long run behavior of the industry}

As above, Doeblin's condition holds here if we set $\phi(\mathscr{A})=P\left\{\mathbf{Y}^{*} \in A \otimes A_{1}\right\}$ for a set $\mathscr{A}$ given by Eq. (2.4). Here $\mathrm{Y}^{*}$ designates a $(2 k+1)$-dimensional vector whose coordinates coincide with first $2 k+1$ coordinates of a generic vector $\mathbf{Y}$ having the same distribution as $\mathbf{Y}^{t}, t \geq 0$. The following result establishes the ergodicity of process Eq. (3.1).

Theorem 3.1. For every set $\mathscr{A}$ given by Eq. (2.4) with probability one

$$
\frac{1}{n} \sum_{t=1}^{n} p^{t}\left(\mathbf{Y}^{0}, \mathscr{A}\right) \rightarrow \pi\left(\mathbf{Y}^{0}, \mathscr{A}\right)
$$

as $n \rightarrow \infty$. Here $\pi\left(\boldsymbol{Y}^{0}, \cdot\right)$ is a stochastic probability measure (since it depends on $\boldsymbol{Y}^{0}$ ), being $\pi_{f}(\cdot)$ for an elementary outcome $\omega \in \Omega$ as long as $\boldsymbol{Y}^{0}$ belongs for this elementary outcome to an ergodic set $£$. Moreover, $p^{t}(\cdot, \cdot)$ designates the transition probability in $t$ steps of process (3.2).

The implications of this theorem in terms of path-dependency (or lack of it) are identical to those discussed above with reference to Theorem 2.1.

Theorem 3.1 implies that for every uniformly bounded characteristic of the industry its time averages converge with probability one to a limit which is a deterministic function of the initial state in the sense given above.

Let us now show that the total productive capacity of the industry is uniformly bounded. Since the minimal size of a firm is bounded by the death threshold, this implies uniform boundedness of the total number of firms in business (if $\varepsilon>0$ ). Hence, we shall be able to derive relations similar to those given in Winter et al. (1997) on convergence of time averages regarding some important characteristics of the industry.

Set $\bar{Q}=H^{-1}(d v C / 2 \lambda)$ and $\hat{Q}=\max (\bar{Q}, 2 k h / C d)$, where $H^{-1}(\cdot)$ designates the inverse function.

Lemma 3.1. With certainty $Q_{t} \leq Q_{*}$ for $t \geq 1$, where $Q_{*}=\hat{Q}[1+\lambda H(0) / v C]+k h / C$.

Proof. Notice that $Q_{1} \leq k h / C \leq Q^{*}$. Eq. (3.1) and the assumption concerning the entry process imply that

$$
Q_{t+1} \leq Q_{t}\left[1-d+\frac{\lambda}{v C} H\left(Q_{t}\right)\right]+\frac{k h}{C}, \quad t \geq 1
$$

If $Q_{t} \geq \hat{Q}$ for some $t \geq 1$, we get that $Q_{t+1} \leq \hat{Q}$. Otherwise, if $Q_{t}<\hat{Q}$,

$$
Q_{t+1} \leq Q_{t}\left[1+\frac{\lambda}{v C} H\left(Q_{t}\right)\right]+\frac{k h}{C}<\hat{Q}\left[1+\frac{\lambda}{v C} H(0)\right]+\frac{k h}{C} .
$$

The lemma is proved.

As a simple consequence of Theorem 3.1 and Lemma 3.1 we have the following result. 
Corollary 3.1. With probability one

$$
\frac{1}{n} \sum_{t=1}^{n} Q_{t} \rightarrow \int_{\tilde{R} ⿱ 亠 乂} \sum_{i=1}^{\infty} \sum_{p=1}^{k} y_{k+p}^{i} \mathrm{~d} \pi\left(\mathbf{Y}^{0}, \mathbf{y}\right)
$$

and, if $\varepsilon>0$,

$$
\frac{1}{n} \sum_{t=1}^{n} v_{t} \rightarrow \int_{\tilde{R}+} \sum_{i=1}^{\infty} \sum_{p=1}^{k} \chi_{A_{p}^{i}(\mathbf{y})} \mathrm{d} \pi\left(\mathbf{Y}^{0}, \mathbf{y}\right)
$$

as $n \rightarrow \infty$. Here $v_{t}$ designates the number of firms in business at $t$. Also, for a vector q given by Eq. (2.2)

$$
\tilde{R}_{+}^{\infty}=\left\{q \in R_{+}^{\infty}: \sum_{i=1}^{\infty} \sum_{p=1}^{k} q_{k+p}^{i} \leq Q_{*}\right\} .
$$

The relation $A_{p}^{i}(\mathbf{y})$ is defined as above.

Indeed, the infinite sum involved in the first limit is bounded by $Q_{*}$ by Lemma 3.1. The sum involved in the second limit does not exceed $C Q_{*} / \varepsilon c<\infty$ if $\varepsilon>0$. Let us turn to the death process.

Theorem 3.2. If $\varepsilon>0$, then each firm dies in a finite random time with probability one.

The proof is given in the Appendix. The intuition is the following.

For simplicity let $\varepsilon<1$. (If $\varepsilon=1$, we need a more complicated argument.) Each firm comes with a capital that exceeds $c$. If it dies, at the moment when this happens its capital does not exceed $\varepsilon c$. Since firms with lower variable costs per unit of output have higher investment rates, a notional firm that lives infinitely long would shrink at least $\varepsilon$ times during the lifetime of a generic firm characterized by the lowest variable costs per unit of output at some particular time (which nonetheless dies in a finite time). Consequently, to prove that no firm can live infinitely long, it is enough to show that: (a) the capital of every alive firm is bounded from above by a constant; and (b) for every level of variable costs per unit of output there is an infinite chain of firms with lower variable costs that are coming and dying one after another.

The capital of an alive firm is bounded from above by the total capital of the industry which, in turn, is bounded with certainty. Thus, (a) holds. The capital of an alive firm is bounded from below by the death threshold and the total capital of the industry is bounded with certainty. Hence the total number of alive firms is bounded with certainty. Consequently, starting from a finite random time $\tau$ every newcoming firm dies in a finite time. According to the postulated learning rule, for every given level of variable costs per unit of output, all newcoming firms have lower variable costs starting from a finite random time $\tau^{\prime}$. Thus, from $\max \left(\tau, \tau^{\prime}\right)$ onward we have the chain required by (b).

Interestingly, in this model the total productive capacity can be indefinitely growing if we drop the assumption that $\lim _{x \rightarrow \infty} H(x)=0$. Namely, let $\lim _{x \rightarrow \infty} H(x)=H$ and $\lambda H / v C>d$. Then, every firm whose variable costs are less than 
$H-v C d / \lambda$ will unboundedly grow. Hence, starting from a finite random time with probability one every newcomer will never die, but rather unboundedly grow. The intuition behind this property is the following. As $H(x)$ approaches its asymptotic value, demand elasticities grow and so does the 'carrying capacity' of the market. Correspondingly, selective pressures get weaker. Since output prices have a positive lower bound, if gross margin are high enough (that is if variable costs are low enough) as to sustain positive net investments, then firms which fulfil these conditions will indefinitely survive (and indeed grow), irrespectively of the fact that an infinite number of even more efficient firms will enter thereafter. One will still observe a dynamic on market shares (with all firms having eventually their shares tending to zero), but given an infinitely expanding market, the number of firms will also be allowed to infinitely grow, and mortality will cease to operate as a selection device. Moreover, the total productive capacity of the economy will also grow in the foregoing circumstances faster than $\gamma^{t}$ as $t \rightarrow \infty$ for every $0<\gamma<1-d+\lambda H / v C$ but slower than $(1-d+\lambda H / v C)^{t}$. In these circumstances, $(1-d+\lambda H / v C)^{t}$ establishes the upper bound of all feasible rates of growth, with history selecting among them. Hence, some (bounded) path-dependency property of industrial dynamics reappears, as soon as the size of the market is allowed to endlessly grow.

\subsection{A numerical run of the model}

Let us turn again to an illustration with a computer simulation (for details cf. footnote 6 , above).

The run presented here has the following parametrization: $k=12, v=1, C=2$, $d=0.3, \lambda=0.6, \varepsilon=0.5$. The demand function is $H(x)=4.1667 \exp (-0.1 x)$. The random variable $\zeta$ is uniformly distributed over $[2,6] ; \xi$ is uniformly distributed over $[0,0.01]$, and the capitals of newcoming firms are uniformly distributed over $[0.02,0.04]$. Figs. 11 and 12 present the price dynamics, while Figs. 13 and 14 show

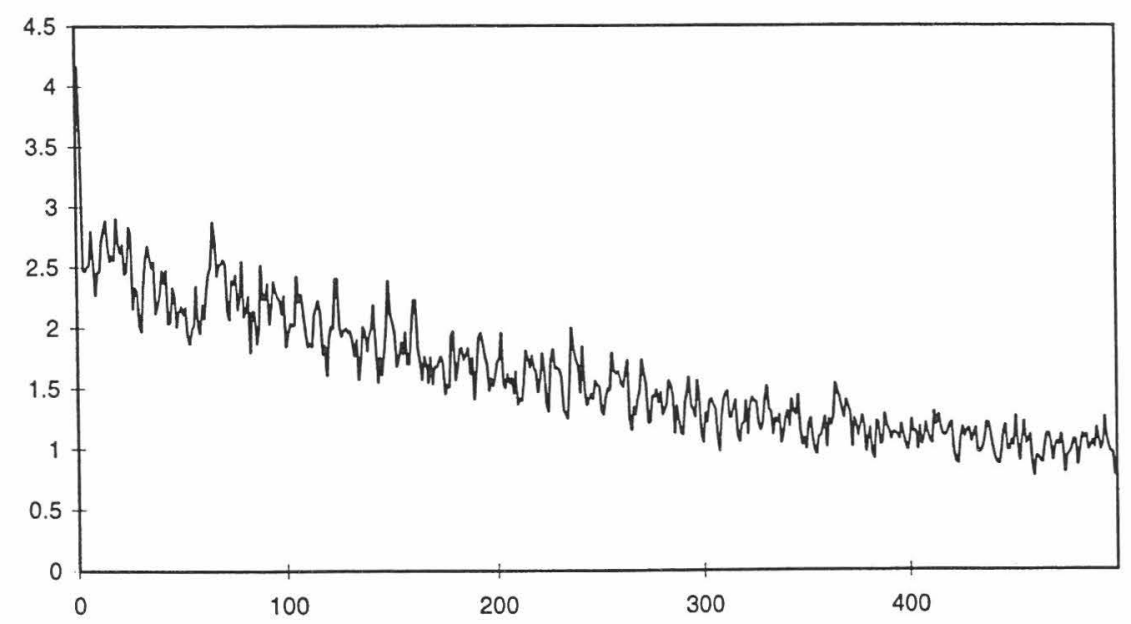

Fig. 11. Price $H\left(Q_{t}\right)$ for $1 \leq t \leq 500$. 


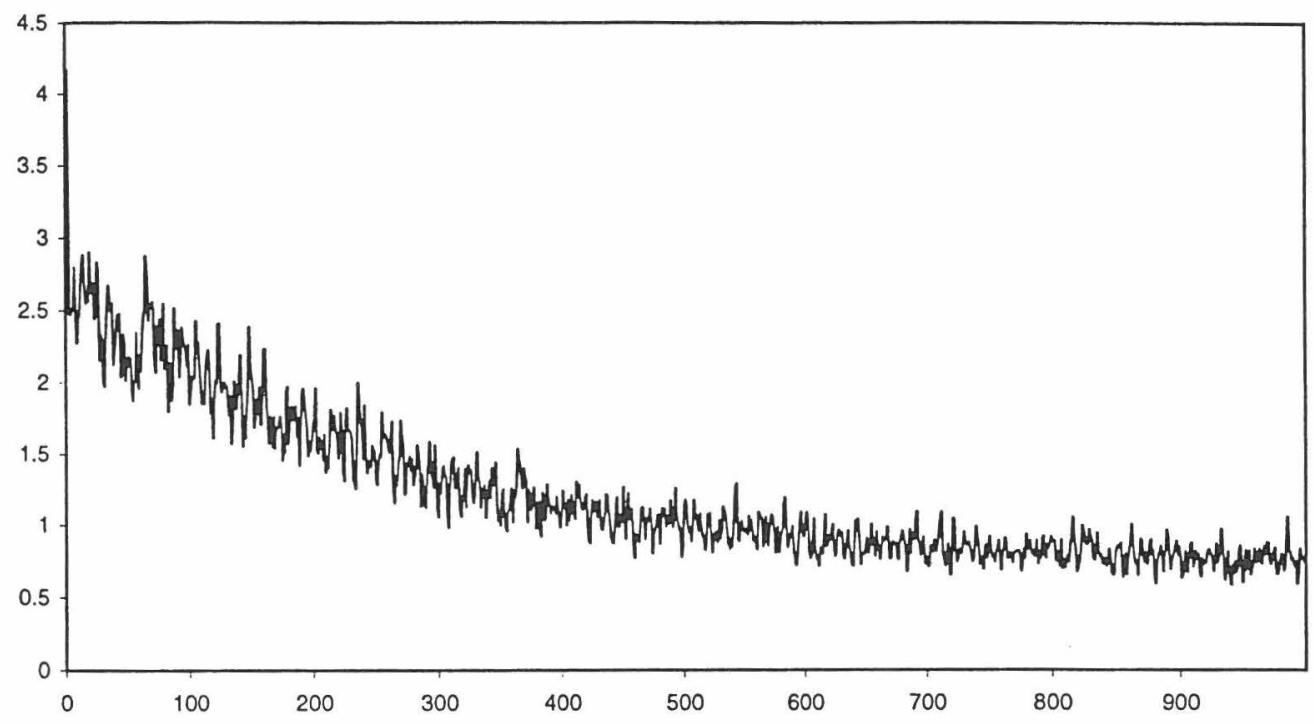

Fig. 12. Price $H\left(Q_{r}\right)$ for $1 \leq t \leq 500$.

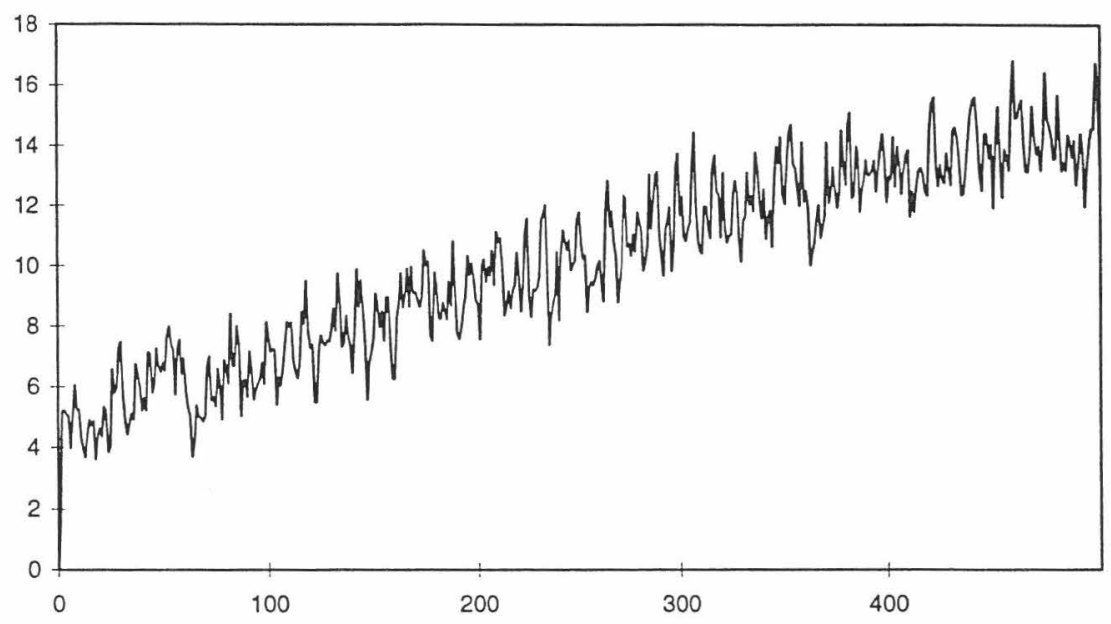

Fig. 13. Total productive capacity $Q$, for $1 \leq t \leq 500$.

for the same time interval the dynamics of the total productive capacity. The evolution of the total number of firms is shown in Figs. 15 and 16, with Figs. 17 and 18 depicting size distributions at $t=50$ and $t=500$. (For prices, productive capacity and number of firms we report also longer simulation runs, with $t=1000$, for a clearer illustration of the long term properties toward which the system tends to converge.) Fig. 19 provides the life time distribution for firms that die before $t=500$. 
Many qualitative properties of the dynamics are similar to those obtained earlier. For example, persistent fluctuations of prices and production capacities and persistent market share turbulence (Fig. 20) are a robust feature of both set-ups. And so are Pareto-type size distributions and skewed age profiles. Interestingly, however, no 'shake-out' seems to occur in the number of firms at some point in its infancy. In this set-up, notwithstanding the property - given appropriate demand condi-

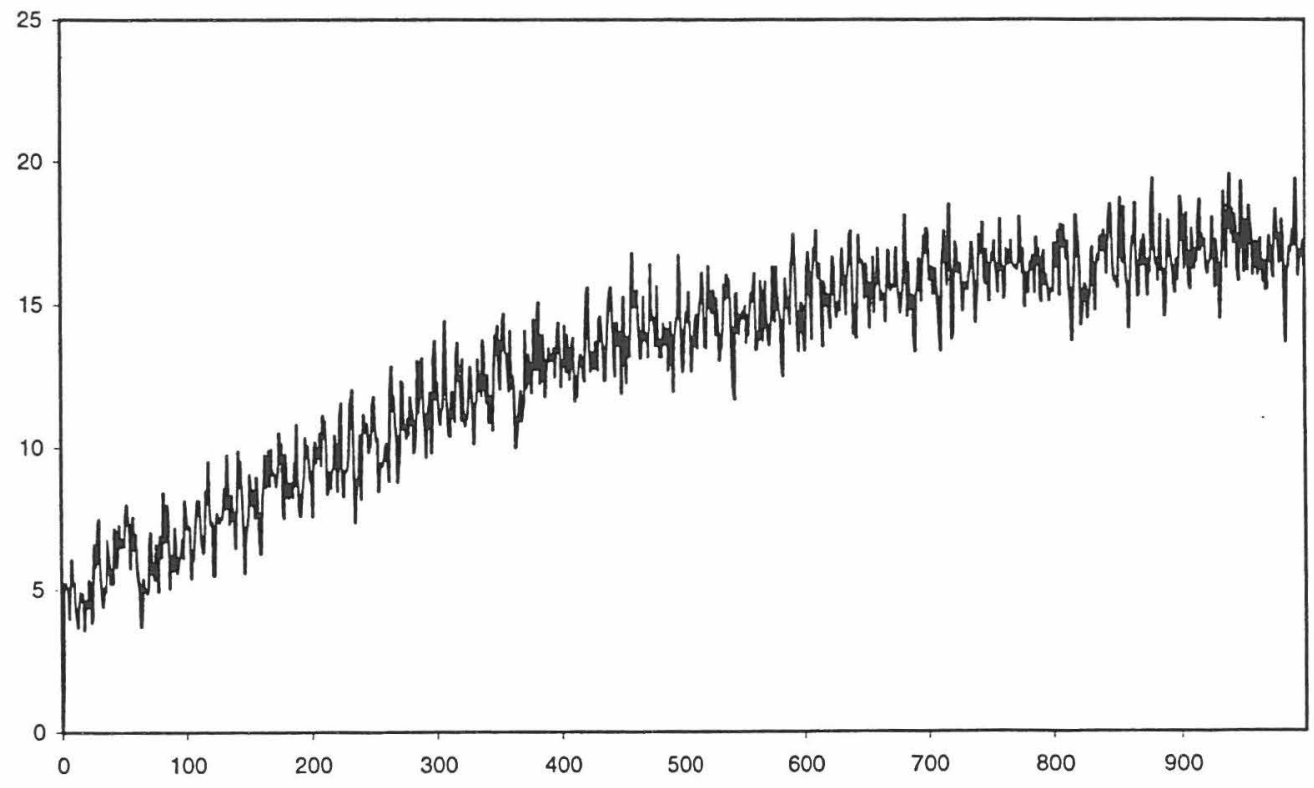

Fig. 14. Total productive capacity $Q_{t}$ for $1 \leq t \leq 1000$.

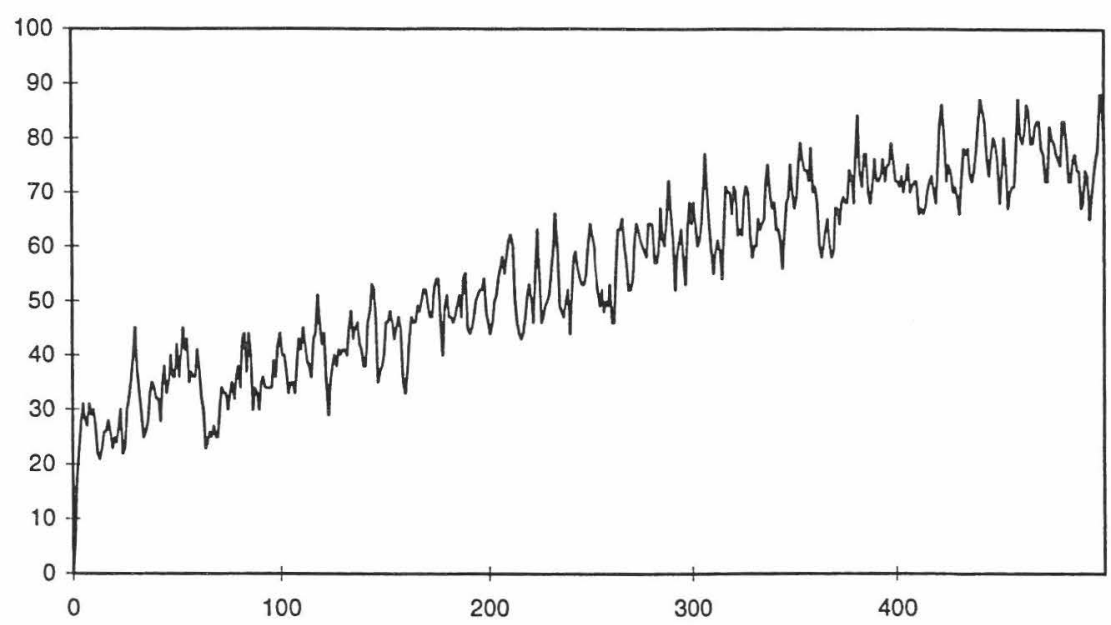

Fig. 15. Total number of firms $0 \leq t \leq 500$. 


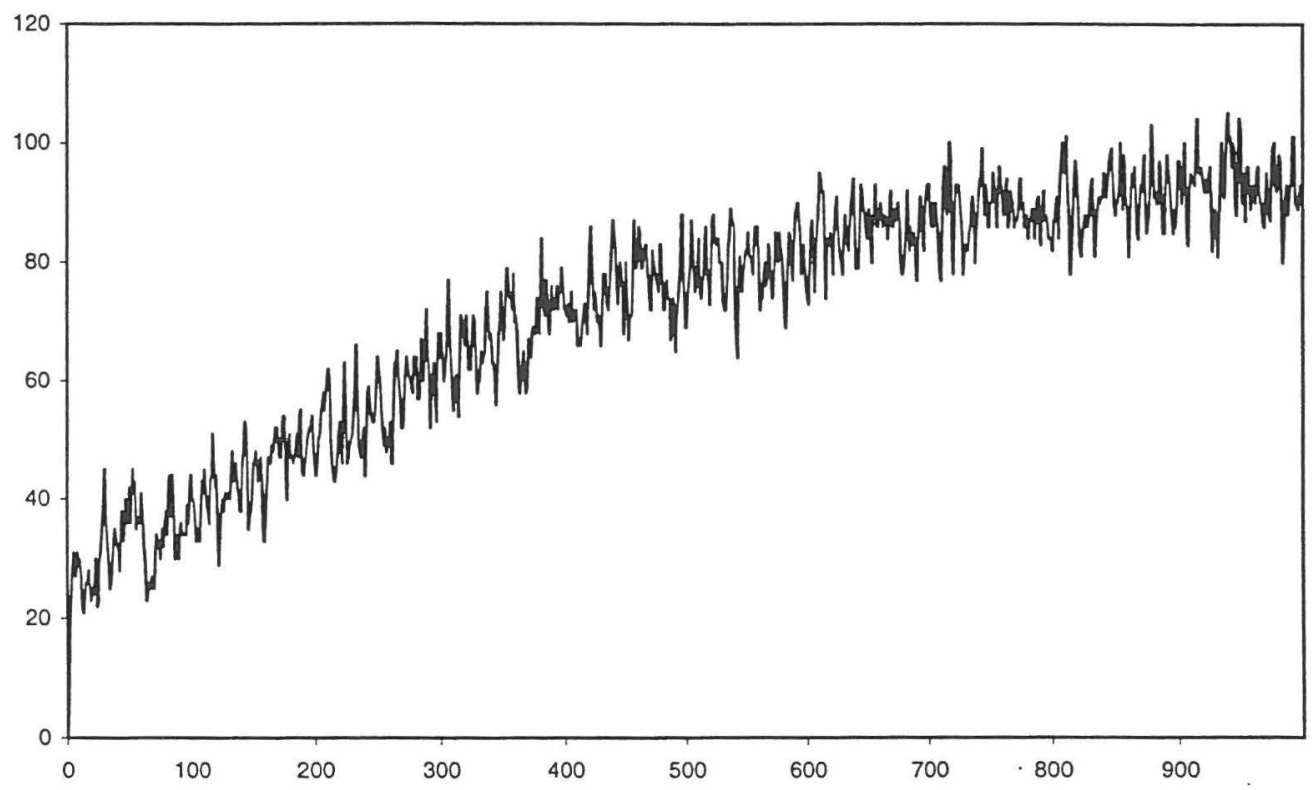

Fig. 16. Total number of firms $0 \leq t \leq 1000$.

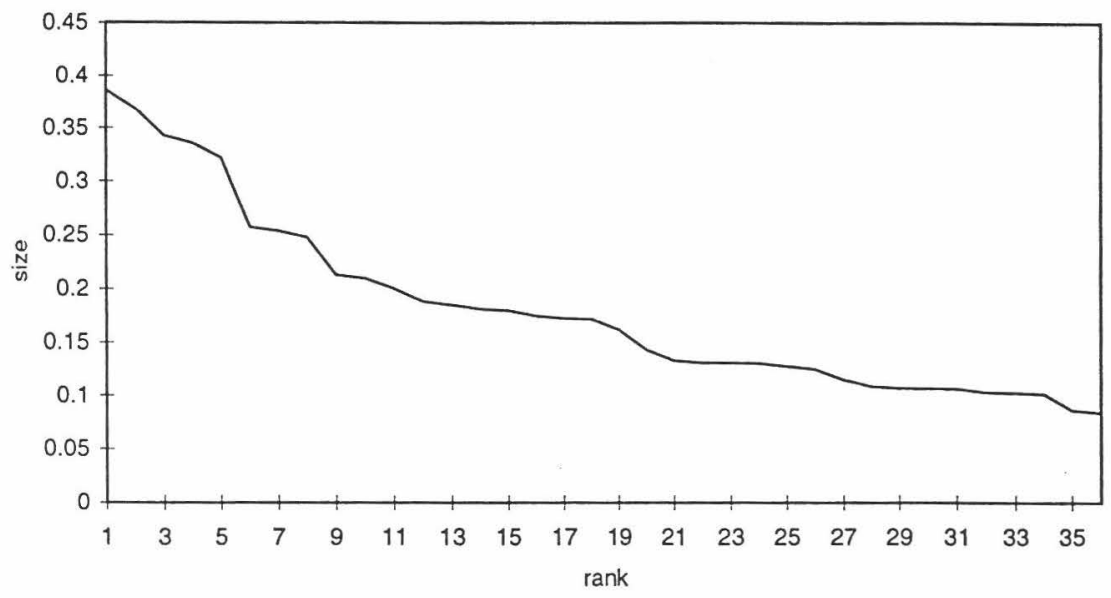

Fig. 17. Size distributions at $t=50$.

tions - that both productive capacity of the industry and the number of firms have upper bounds, the industry seems to approach them without any major structural discontinuities ${ }^{13}$ with concentration falling in the long term (Fig. 21).

\footnotetext{
${ }^{13}$ A similar profile in the evolution of the number of firms is also obtained, under somewhat similar Schumpeter Mark I regimes of learning, in Dosi et al. (1995).
} 


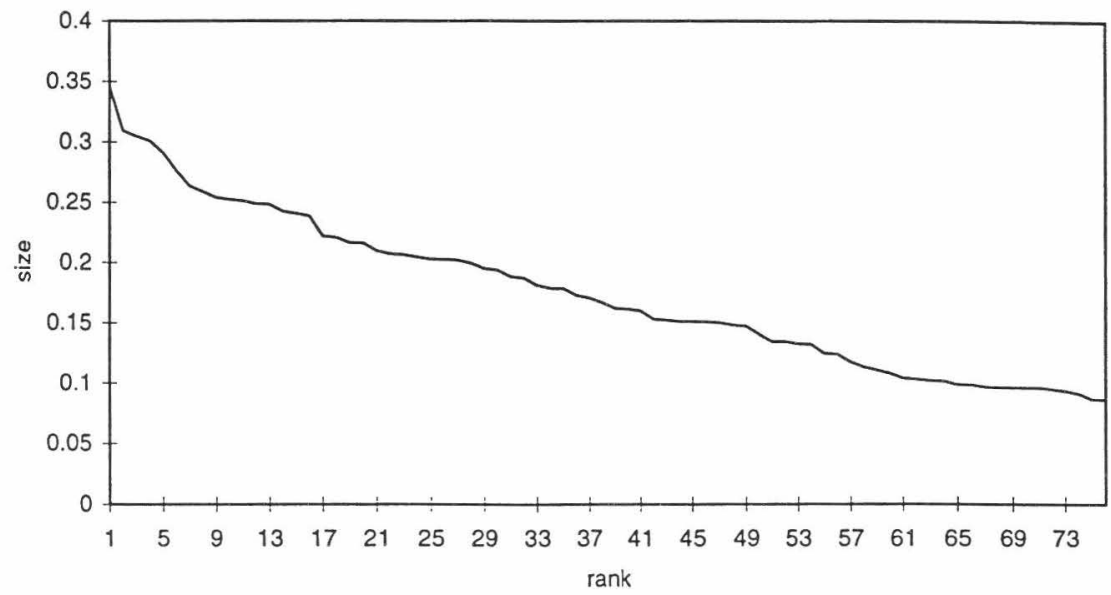

Fig. 18. Size distributions at $t=500$.

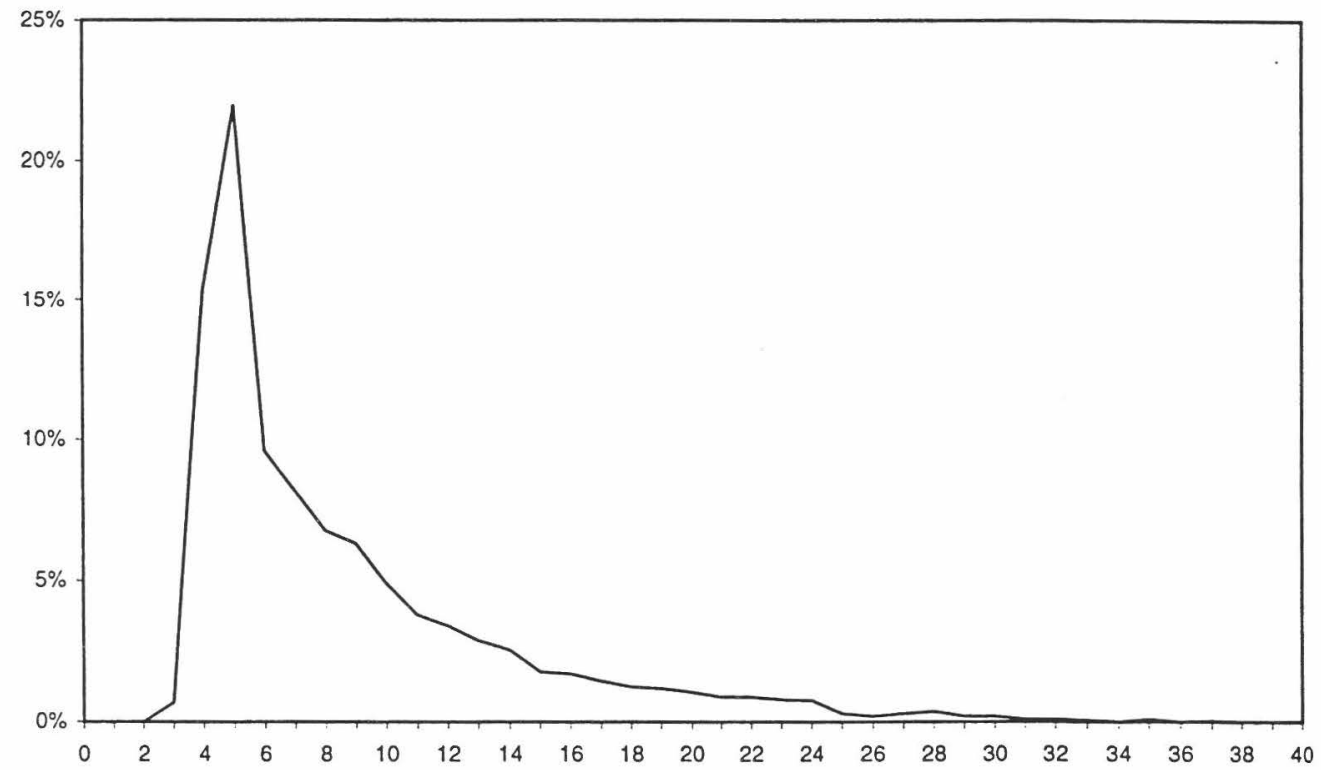

Fig. 19. Life time distribution for $1 \leq t \leq 500$.

\section{Modeling learning on both capital and labour efficiencies}

The two foregoing models may also be combined to account for those (empirically more plausible) circumstances whereby entrants are allowed to innovate, in probability, will respect to both capital and labour efficiencies. In order to define this set-up one needs four random variables: $\xi_{C}, \xi_{L}, \zeta_{C}$, distributed over $\left[a_{C}, b_{C}\right]$, and $\zeta_{L}$, distributed over $\left[a_{L}, b_{L}\right]$. Here $E \xi_{i}>0, D \xi_{i}<\infty$, and $0<a_{i}<b_{i}<\infty, i=C$, $L$. 


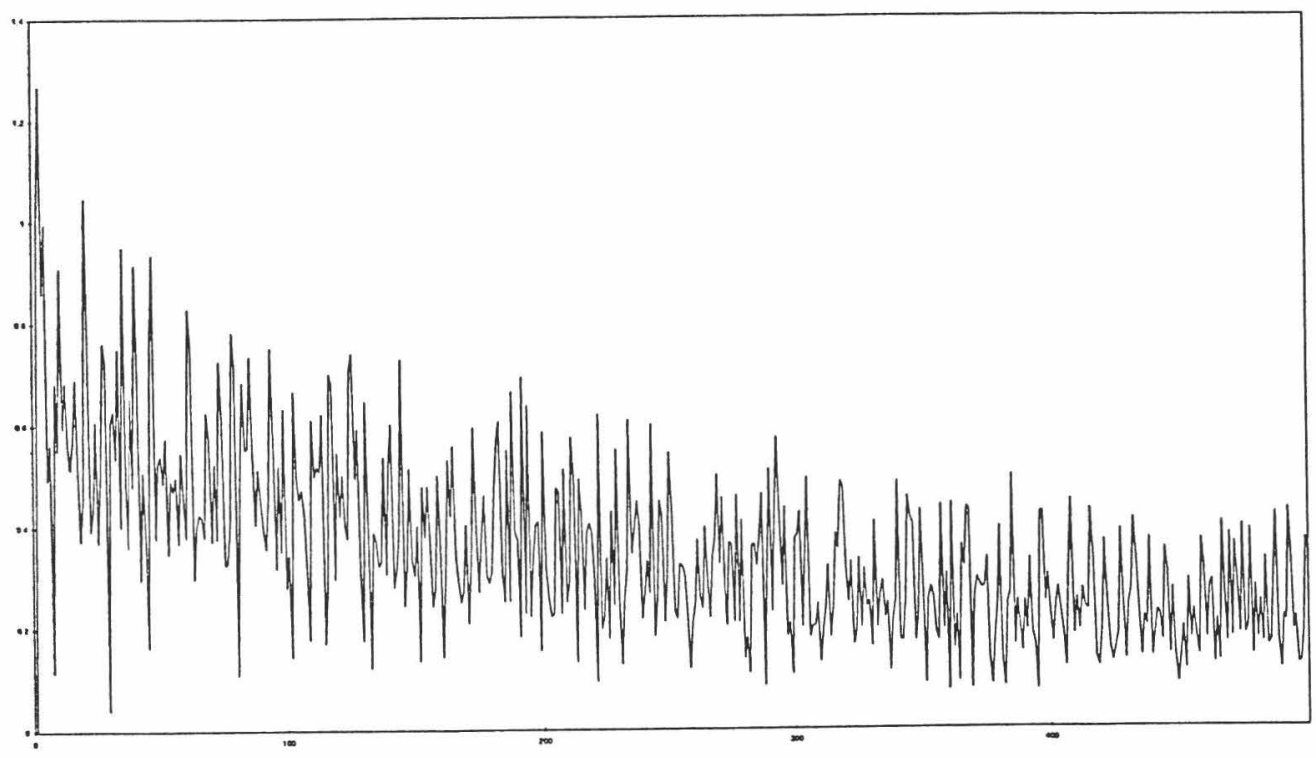

Fig. 20. Turbulence index for $1 \leq t \leq 499$.

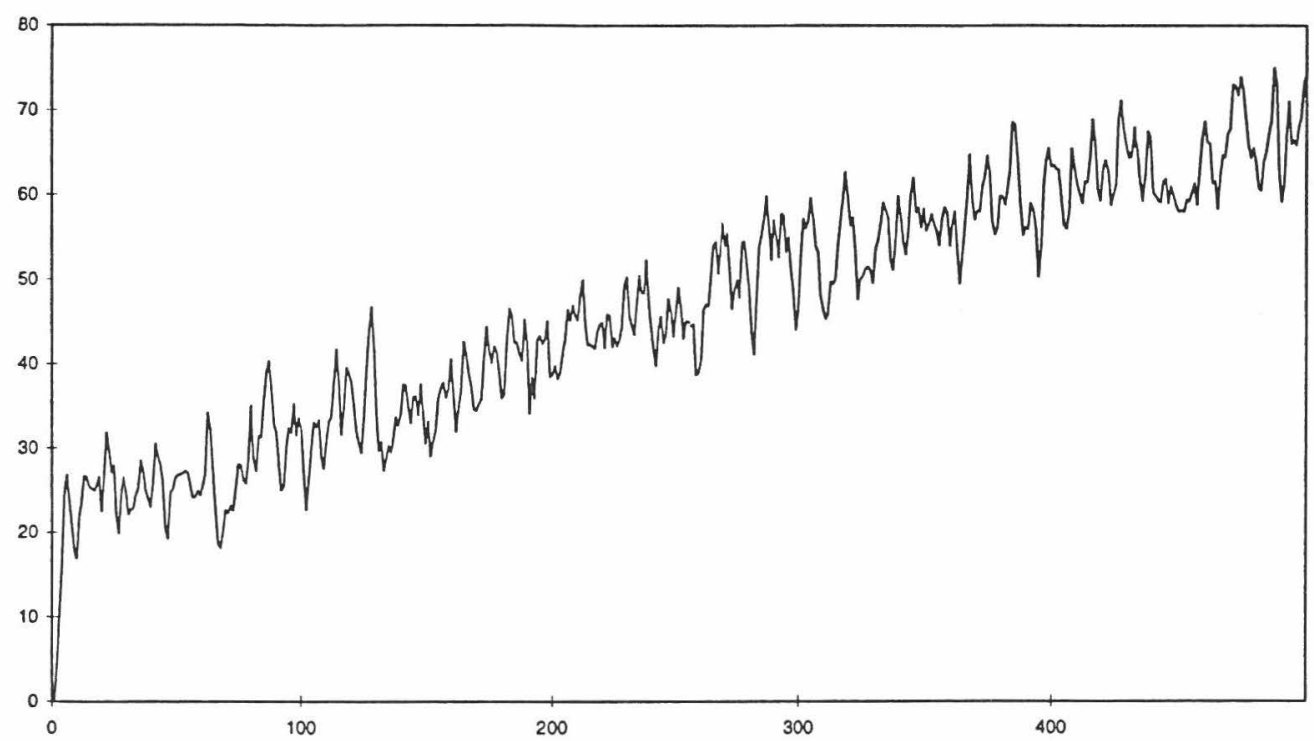

Fig. 21. Equivalent number of firms from the Hirfindhal index for $1 \leq t \leq 500$.

Set, for $t \geq 0$

$$
A_{t+1}^{(C)}=A_{t}^{(C)}+\xi_{C}^{t+1}, \quad A_{0}^{(C)}=0, \quad \text { and } \quad A_{t+1}^{(L)}=A_{t}^{(L)}+\xi_{L}^{t+1}, \quad A_{0}^{(L)}=0 .
$$

Allowing for $k \geq 1$ newcomers at each time, $t \geq 0$ define their capital ratios and variable costs as $\exp \left\{-A_{t}^{(c)}\right\} \zeta_{\mathrm{c}}^{\mathrm{i}}$ and $\exp \left\{-A_{t}^{(L)}\right\} \zeta_{L}^{i}, t k+1 \leq i \leq(t+1) k$. Here $\zeta_{i}^{t}$, 
$t \geq 0, i=C, L$, and $\zeta_{i}^{j}, j \geq 1, i=C, L$, are independent (in all indexes) realizations of the corresponding random variables.

For a firm $i$ (whose capital ratio is $a_{i}$ and variable costs are $m_{i}$ ) manufacturing at time $t$ we have as above

$$
Q_{t+1}^{i}=Q_{t}^{i}\left\{1-d+\frac{\lambda}{v a_{i}} \max \left[H\left(Q_{t}\right)-m_{i}, 0\right]\right\} X_{Q i}\left\{1-d+\frac{\lambda}{v a_{i}} \max \left[H\left(Q_{t}\right)-m_{i}, 0\right]\right\} \geq \tau c / a_{i}
$$

Interestingly, in this set-up productive capacities of newcomers grow to infinity in the same way as in the model with increasing productivity alone. So unboundedly grows the total productive capacity of the industry. Hence, the limit behavior of this industry turns out to be similar to the growth pattern of an industry where newcomers learn how to improve the productivity of capital alone, as in the first of the foregoing models.

\section{Conclusions}

In this work we have explored some dynamic properties of industrial dynamics driven by an ever-lasting flow of entrants which might, in probability, be carriers of technological innovations (that is, in our simple model, more efficient techniques of production).

Some properties of the ensuing industrial dynamics appear to be generic features of a wide class of evolutionary processes nested into microeconomic heterogeneity and market selection. In particular, (a) persistent fluctuations of aggregate variables - such as price, production capacity, total output - ; (b) turbulence in market shares; and (c) skewed size distributions of firms appear to be robust features of the competitive process, irrespectively of any more detailed characterization of the origins and the bounds upon microeconomic heterogeneity. (In this respect compare the results presented here with Winter et al., 1997.) Other properties corresponding to other empirically observable regularities - depend, on the contrary, upon more specific characterizations of the ways micro heterogeneity is generated. That includes whether and how innovations are generated along the history of the industry.

First, and most intuitively, necessary (but not sufficient) condition for the industry to exponentially grow is the persistent enlargement of notional opportunities of innovation. In the foregoing model the process is represented as an endogenous drift in the set of input coefficients stochastically attainable at each time, conditional on the best-practise knowledge already achieved at such a time. It is an 'open-ended' dynamic insofar as, in the limit, there is no bound upon the possibilities of discovery, even if at each time what is attainable is ultimately constrained by what has been learned up to that time.

Second, as just mentioned, such open-endness in innovative opportunities is not sufficient to guarantee self-sustained growth. Rather, the latter stems from the interplay between learning opportunities and demand patterns. A significant implication of the foregoing modeling experiments - where, on purpose, we did not 
allow any exogenous demand drift - is that notionally unbounded dynamic increasing returns may fully exert their impact upon output growth only insofar as they are not limited by the extent of the market, to paraphrase the old adagio by Adam Smith. In the set-up with learning about capital efficiency, the market indefinitely grows in real terms because technical progress provides, for its nature, also a corresponding possibility of expansionary investment in productive capacity. Given the hypotheses of that specification of the model, even if the demand curve does not shift (in nominal terms) over time, capital costs of production per unit of output progressively wither away as time goes on, and, as a consequence, the benefits of increasing returns to knowledge accumulation can be fully reaped throughout.

Conversely, this might not be the case with learning occuring only with respect to labour efficiencies. Here, the long-term evolutionary outcomes depend upon the interplay between the shape of the demand curve and the level of fixed capital costs per unit of output. The latter obviously set a ceiling to the maximum expansion of production capacity from any $t$ to $t+1$ for whatever gross margin each firm is able to obtain. Whether such a ceiling to micro growth in any finite time carries over to the long-run system properties is, however, a quite different matter. As discussed above, under these circumstances, self-sustained growth of the industry can be attained only if the shape of the demand curve is such as to allow in the long-run an indefinite expansion of total gross surplus and of net investments in production capacity ${ }^{14}$.

More generally, as both our analytical results and simulations show, the long-run dynamics of the industry depends also on the interplay between patterns of technological learning and demand conditions (this is a point emphasized also in more static set-ups by Sutton, 1998, which turns out to apply in our model even when disposing of any assumption of 'rational' consistency amongst microbehaviors).

In this paper we focused upon a specific archetype of learning dynamics, which — in tune with earlier literature - we called Schampeter Mark I. In such a stylized learning regime, one restricted a positive probability of learning to entrants, with inputs coefficients fixed thereafter for all incumbents. While an obvious violence to a much more messy empirical evidence, this modeling framework allows an easier identification of the properties of that subset of learning processes whereby incumbent knowledge is highly inertial and the dominant source of change is the arrival of new entrepreneurial trials.

Given the formal Schumpeter Mark I set-up, we show as our third major conclusion that generally the process of competition and collective growth must be fueled by an unending process of entry and exit, with each individual firm dying with probability one in finite time and, whereby, paraphrasing Geroski, the life of each firm tends to be 'nasty, brutish and short' - cf. Geroski and Schwalbach (1991) and Geroski (1995). (The only exception we find is under some rather special

\footnotetext{
${ }^{14}$ Clearly, the condition would be more easily met if one allowed some positive drift over time in demand curves themselves (and plausibly also negative drifts for 'shrinking' industries).
} 
demand patterns whereby an infinitely growing number of firms can survive, with non-decreasing absolute size, notwithstanding vanishingly small market shares, given exponentially growing markets).

Fourth, with the open-ended innovative dynamics considered here, the role of history, i.e. more formally, path-dependence - more forcefully appears in the account of long-term dynamics. As already noted in Winter et al. (1997). even in a 'closed' world of technological options, the expressions for long-term average statistics for the industry contains a possible dependence upon initial conditions (insofar as more than one ergodic set exists, determining the Markovian structure of industry evolution(s)). Here, however, path-dependency acquires much more straightforward implications. In essence, under all conditions whereby the industry unboundedly grows, what one is able to prove, in a history-independent fashion, is that a whole class of exponential functions may fit any pattern generated under these conditions. However, as we show above, path-dependence essentially affects which growth rate turns out to be selected also in the long-term.

\section{Acknowledgements}

We are grateful to Andrea Bassanini, the participants to the Conference on Economic Models of Evolutionary Dynamics with Interacting Agents (The Abdus Salam International Centre for Theoretical Physics, Trieste, Italy, September 1998) and two anonymous referees for their comments on previous drafts. Mariele Bertè and Marco Valente have been of crucial help for the simulation of the model. Francesca Chiaromonte preciously contributed to the endless discussions concerning both the fine details of the model and its computer simulations.

We thank the International Institute for Applied Systems Analysis, the Fujitsu Research Institute for Advanced Information (Japan), the University of Trento and the European Union (ESSY Project, TSER, DG XII) which at various stages supported the research leading to this work. Usual caveats apply.

\section{Appendix A}

In the following, we provide the proofs omitted in the text.

Lemma 2.1. The total productive capacity $Q_{1}$ of the industry goes to infinity with probability one as $t \rightarrow \infty$.

Proof. We have to show that for every $\delta \in(0,1)$ and every positive $Q$ there is a finite time instant $t(\delta, Q)$ such that

$$
P\left\{Q_{t} \geq Q, t \geq t(\delta, Q)\right\} \geq 1-\delta .
$$

By the strong law of large numbers (see, for example, Loève, 1955, p. 239) 


$$
A_{t}=(t+1)\left[E \xi+o_{t}^{1}(1)\right] .
$$

Here $E \xi$, designates the expected value of $\xi$. Also, $o_{t}^{i}(1) \rightarrow 0$ with probability one as $t \rightarrow \infty$. Fix a $\delta \in(0,1)$ and a $Q \in(0, \infty)$. Since $o_{t}^{1}(1) \rightarrow 0$ with probability one, there is a finite time instant $t(\delta)$ such that

$$
P\left\{\left|o_{t}^{1}(1)\right|<\frac{1}{2} E \xi, t \geq t(\delta)\right\} \geq 1-\delta .
$$

This implies

$$
P\left\{A_{t} \geq \frac{t+1}{2} E \xi, t \geq t(\delta)\right\} \geq 1-\delta .
$$

Hence, capital ratios of firms coming from $t(\delta)$ onward do not exceed $\exp \{-t+1 / 2 E \xi\} b$ with probability $1-\delta$. Since initial capitals are always larger than $c$, the total productive capacity $V_{t}=\exp \left\{A_{t}\right\} \Theta^{t}$ of all firms coming at $t \geq t(\delta)$, exceeds $\exp \{[(t+1) / 2] E \xi\} k c / b$ with probability $1-\delta$. Here

$$
\Theta^{t}=\sum_{i=1}^{k} \theta^{t k+i} / \zeta^{t k+i}
$$

Notice that

$$
Q_{t+1} \geq V_{t}, \quad t \geq 0 .
$$

Hence,

$$
P\left\{Q_{t} \geq \exp \left\{\frac{t}{2} E \xi\right\} k c / b, t \geq t(\delta)\right\} \geq 1-\delta .
$$

Set $t_{Q}=\min t: \exp \{(t / 2) E \xi\} k c / b \geq Q$. For $t(\delta, Q)=\max \left[t(\delta), t_{Q}\right]$ inequality (a1) holds true.

The lemma is proved.

Theorem 2.2. If $\varepsilon>0$, then every firm dies in a finite random time with probability one.

Proof. Assume on the contrary that there is a firm surviving infinitely long with positive probability. Designating by $q_{t}$ its capital at time $t$, we have

$$
P\left\{q_{1} \geq \varepsilon \mathcal{E}, t \geq t_{0}\right\}>\delta>0 .
$$

Here $t_{0} \geq 0$ stands for the time instant when the firm came to the industry. Also, take into account here that a firm stays alive as long as its capital does not drop below the death threshold $\varepsilon c$.

Let $Q=\min x>0: 1-d+\lambda H(x) / v a_{*} \leq 1-d / 2$, where $a_{*}$ designates the capital per unit of output of the firm surviving infinite time. By Lemma 2.1 there is a finite time instant $t(\delta / 2, Q)$ such that

$$
P\{Q, \geq Q, t \geq t(\delta / 2, Q)\} \geq 1-\delta / 2 .
$$

For every pair of events $A, B \in \mathscr{F}$ 


$$
P\{A \cap B\}=P\{A\}-P\{A \cap(\Omega \backslash B)\} \geq P\{A\}-P\{\Omega \backslash B\} .
$$

Taking this into account, we have by (a4) and (a5) that

$$
P\left\{Q_{t} \geq Q, q_{t} \geq \varepsilon c, t \geq t_{1}\right\} \geq P\left\{Q_{t} \geq Q, t \geq t_{1}\right\}-1+P\left\{q_{t} \geq \varepsilon c, t \geq t_{1}\right\}>\delta / 2,
$$

where $t_{1}=\max \left[t_{0}, t(\delta / 2, Q)\right]$. Thus, the event $\left\{Q_{t} \geq Q, q_{t} \geq \varepsilon c, t \geq t_{1}\right\}$ occurs with positive probability. But, if it happens, then simultaneously

$$
q_{t+1} \leq q_{t}(1-d / 2) \quad \text { and } \quad q_{t} \geq \varepsilon c
$$

for $t \geq t_{1}$. These inequalities cannot hold simultaneously. Indeed, $q_{t_{1}}$ is a finite value, namely,

$$
q_{t_{1}}=q_{t_{0}} \prod_{i=t_{0}+1}^{t_{1}-1}\left[1-d+\frac{\lambda}{v} H\left(Q_{i}\right) / a_{*}\right] .
$$

Since $1-d / 2 \in(0,1)$, the first inequality implies that from $t_{1}$ onward the sequence $\left\{q_{t}\right\}$ is exponentially declining. The second inequality assumes that this sequence is uniformly bounded from below by $\varepsilon c>0$. This contradiction shows that there cannot be a firm surviving infinitely long with positive probability.

The theorem is proved.

Theorem 2.3. With probability one $\exp \{-\alpha t\} Q_{t} \rightarrow \infty$ as $t \rightarrow \infty$ for every $\alpha<E \xi$. Moreover, if

$$
\lim _{x \rightarrow \infty} H(x) x=0,
$$

then with probability one $\exp \{-\alpha t\} Q_{t} \rightarrow 0$ as $t \rightarrow \infty$ for every $\alpha>E \xi$.

Proof. The first statement holds by Eq. (a3). Let us prove the second one. Notice that

$$
Q_{t+1}=Q_{t}\left[1-d+\frac{\lambda}{v} H\left(Q_{t}\right) \sum_{i=1}^{n_{t}} Q_{t}^{i} / Q_{t} a_{i}\right]+V_{t}-\mathscr{E}_{t}, \quad t \geq 1 .
$$

Recall that $a_{i}$ stands for the capital per unit of output of the $i$-th firm. Also. $\mathscr{E}_{t} \geq 0$ designates the total outflow of productive capacity at $t$ due to mortality of inefficient firms. Dropping $\mathscr{E}_{t}$ in Eq. (a7), we get

$$
Q_{t+1} \leq Q_{t}\left[1-d+\frac{\lambda}{v} H\left(Q_{t}\right) \sum_{i=1}^{n_{t}} Q_{t}^{i} / Q_{t} a_{i}\right]+V_{t}, \quad t \geq 1 .
$$

Since $H(\cdot)$ decreases, by Eq. (a3) this inequality can be further relaxed

$$
Q_{t+1} \leq Q_{t}\left[1-d+\frac{\lambda}{v} H\left(V_{t-1}\right) \sum_{i=1}^{n_{t}} Q_{t}^{i} / Q_{t} a_{i}\right]+V_{t}, \quad t \geq 1 .
$$

We have that

$$
\sum_{i=1}^{n_{t}} Q_{t}^{i} / Q_{t} a_{i} \leq \frac{1}{\min _{i=1,2, \ldots, n_{t}} a_{i}}, \quad \min _{i=1,2, \ldots, n_{t}} a_{i} \geq \exp \left\{-A_{t-1}\right\} a,
$$




$$
V_{t} \geq \exp \left\{A_{t}\right\} k c / b .
$$

Consequently,

$$
\sum_{i=1}^{n_{t}} Q_{t}^{i} / Q_{t} a_{i} \leq \frac{b}{k c a} V_{t-1}
$$

Thus, by Eq. (a8), we get

$$
Q_{t+1} \leq Q_{t}\left[1-d+\frac{\lambda b}{k v c a} H\left(V_{t-1}\right) V_{t-1}\right]+V_{t}, \quad t \geq 1 .
$$

By Eq. (a2), $V_{t} \rightarrow \infty$ with probability one as $t \rightarrow \infty$. Hence, taking into account that $x H(x) \rightarrow 0$ as $x \rightarrow \infty$, the latter inequality can be rewritten as

$$
Q_{t+1} \leq Q_{t}\left[1-d+o_{t}^{2}(1)\right]+V_{t}, \quad t \geq 1,
$$

where $o_{t}^{2}(1) \rightarrow 0$ with probability one as $t \rightarrow \infty$.

Fix an $\alpha>E \xi$,. Setting $X_{t}=\exp \{-\alpha t\} Q_{t}$ and $W_{t}=\exp \{-\alpha(t+1)\} V_{t}$, we get by Eq. (a9) that

$$
X_{t+1} \leq \exp \{-\alpha\} X_{t}\left[1-d+o_{t}^{2}(1)\right]+W_{t} \leq X_{t}\left[1-d+o_{t}^{2}(1)\right]+W_{t}, \quad t \geq 1 .
$$

We have to show that for every $\delta>0$ and $\sigma \in(0,1)$ there is a finite time instant $t(\delta, \sigma)$ such that

$$
P\left\{X_{t} \leq \delta, t \geq t(\delta, \sigma)\right\} \geq 1-\sigma .
$$

Fix some $\delta>0$ and $\sigma \in(0,1)$. Since $o_{t}^{1}(1)$ in Eq. (a2) converges to zero with probability one, there is a finite time instant $t_{1}(\sigma)$ such that

$$
P\left\{\left|o_{t}^{1}(1)\right| \leq \frac{1}{2}(\alpha-E \xi), t \geq t_{1}(\sigma)\right\} \geq 1-\sigma / 2 .
$$

Similarly, there is a finite time instant $t_{2}(\sigma)$ such that

$$
P\left\{\left|o_{t}^{2}(1)\right| \leq \frac{d}{2}, t \geq t_{2}(\sigma)\right\} \geq 1-\sigma / 2 .
$$

By Eqs. (a2) and (a12) we conclude that

$$
P\left\{W_{t} \leq \frac{k h}{a} \exp \left\{-\frac{(\alpha-E \xi)(t+1)}{2}\right\}, t \geq t_{1}(\sigma)\right\} \geq 1-\sigma / 2 .
$$

Thus, setting $t(\sigma)=\max \left[t_{1}(\sigma), t_{2}(\sigma)\right]$ and taking into account Eqs. (a10), (a13) and (a14), we get by Eq. (a6) that

$$
P\left\{X_{t+1} \leq X_{t}(1-d / 2)+\frac{k h}{a} \exp \left\{-\frac{(\alpha-E \xi)(t+1)}{2}\right\}, t \geq t(\sigma)\right\} \geq 1-\sigma,
$$

or, equivalently, for every finite $n \geq 1$

$$
\begin{aligned}
& P\left\{X_{t+n} \leq X_{t}(1-d / 2)^{n}+\frac{k h}{a} \exp \left\{-\frac{(\alpha-E \xi)(t+1)}{2}\right\} \sum_{i=0}^{n-1}(1-d / 2)^{n-i-1}\right. \\
& \left.\times \exp \left\{-\frac{(\alpha-E \xi) i}{2}\right\}, t \geq t(\sigma)\right\} \geq 1-\sigma .
\end{aligned}
$$


Since

$$
\begin{aligned}
& \sum_{i=0}^{n-1}(1-d / 2)^{n-i-1} \exp \left\{-\frac{(\alpha-E \xi) i}{2}\right\} \leq \sum_{i=0}^{n-1}(1-d / 2)^{n-i-1} \\
& =\frac{2}{d}\left[1-(1-d / 2)^{n}\right]<\frac{2}{d},
\end{aligned}
$$

the latter unequality implies that for every finite $n \geq 1$

$$
P\left\{X_{t+n} \leq X_{t}(1-d / 2)^{n}+\frac{2 k h}{a d} \exp \left\{-\frac{(\alpha-E \xi)(t+1)}{2}\right\}, t \geq t(\sigma)\right\} \geq 1-\sigma .
$$

There is a finite $t_{\delta}$ such that for $t \geq t_{\delta}$

$$
\frac{2 k h}{a d} \exp \left\{-\frac{(\alpha-E \xi)(t+1)}{2}\right\} \leq \delta / 2 \text {. }
$$

Hence, by the previous inequality we get that for every finite $n \geq 1$

$$
P\left\{X_{t+n} \leq X_{t}(1-d / 2)^{n}+\delta / 2, t \geq t_{\delta, \sigma}\right\} \geq 1-\sigma,
$$

where $t_{\delta, \sigma}=\max \left[t(\sigma), t_{\delta}\right]$. By Eq. (a7), the random variable $Q_{t_{j, \sigma}}$ is finite with certainty. Hence, $X_{t \delta \sigma}$ is also a finite random variable with certainty. There is a finite number $n_{\delta, \sigma}$ such that

$$
P\left\{X_{t_{\delta, \sigma}}(1-d / 2)^{n} \leq \delta / 2, n \geq n_{\delta, \sigma}\right\}=1 .
$$

Setting $t(\delta, \sigma)=\dot{t}_{\delta, \sigma}+n_{\delta, \sigma}$, by Eqs. (a15) and (a16) we get Eq. (al1).

The theorem is proved.

Next, let us show that in the version of the model with growing labor productivity each firm dies in a finite random time with probability one.

Theorem 3.2. If $\varepsilon>0$, then each firm dies in a finite random time with probability one.

Proof. The death threshold implies that if a firm lives infinitely long, then its capital does not drop below $\varepsilon c$. Since the total productive capacity of the industry is bounded with certainty, we conclude that starting from a finite random time $r$ with probability one every newcoming firm dies in a finite time. Indeed, otherwise we would have infinitely many firms living infinitely long. This, by boundness from below of their capitals, would imply that the total productive capacity goes to infinity.

At time $t \geq \tau$ consider two firms: one with capital $c_{t}$ and variable costs per unit of output $m$, the other with capital $c_{t}^{\prime}$ and variable costs per unit of output $m^{\prime}$, $m>m^{\prime}$. Then 


$$
\frac{c_{t+1}}{c_{t+1}^{\prime}}=\frac{c_{t}\left\{1-d+\frac{\lambda}{v C} \max \left[H\left(Q_{t}\right)-m, 0\right]\right\} \chi_{c_{t}\left\{1-d+\frac{\lambda}{v C} \max \left[H\left(Q_{t}\right)-m, 0\right]\right\} \geq \varepsilon c}}{c_{t}^{\prime}\left\{1-d+\frac{\lambda}{v C} \max \left[H\left(Q_{t}\right)-m^{\prime}, 0\right]\right\} \chi_{c_{t}}\left\{1-d+\frac{\lambda}{v C} \max \left[H\left(Q_{t}\right)-m^{\prime}, 0\right]\right\} \geq \varepsilon c} .
$$

Assume that there is a firm living infinitely long with positive probability. Set $c_{t}$ for its capital at $t$ and $m$ for its variable costs per unit of output. Then

$$
P\left\{c_{t} \geq \varepsilon c, t \geq \tau^{\prime}\right\}=\delta>0,
$$

where $\tau^{\prime}<\tau$ stands for the time instant when it comes to the industry. By Eq. (a2) there is a time instant $t_{1}$ such that

$$
P\left\{A_{t}>\frac{t+1}{2} E \xi, t \geq t_{1}\right\} \geq 1-\delta / 2 .
$$

Choose $t_{2}$ such that $\exp \{[(t+1) / 2] E \xi\} h<m$ for $t \geq t_{2}$. Then with probability exceeding $1-\delta / 2$ every firm coming after $t^{*}=\max \left(t_{1}, t_{2}\right)$ has variable costs less than $m$. Consider a time instant $t \geq \max \left(t^{*}, \tau\right)$. There is a firm coming at $t$. Set $c_{t}^{\prime}$ for its capital and $m^{\prime}$ for its variable costs. Since we are in the time domain where every entrant dies in a finite time, this new firm dies at a finite time instant $t^{\prime}>t$ with probability one. Since $m^{\prime}<m$, by Eq. (a17) we get that

$$
c_{t^{\prime}} \leq c_{t} \frac{c_{t^{\prime}}^{\prime}}{c_{t}} \leq c_{t} \frac{\varepsilon c}{c} \leq \varepsilon c_{t^{\prime}} .
$$

At $t^{\prime}$ another firm comes to the industry and, again, its variable costs are less than $m$. Similarly, it dies at $t^{\prime \prime}$ and we obtain that $c_{t^{\prime \prime}} \leq \varepsilon c_{t^{\prime}}$ or $c_{t^{\prime \prime}} \leq \varepsilon^{2} c_{t}$.

Let $\varepsilon<1$. Since $c_{t} \leq C Q_{*}$ for $t \geq \tau^{\prime}$, we conclude that $c_{t_{k}} \rightarrow 0$ as $k \rightarrow \infty$ for some sequence of finite time instants $t_{k}, k \geq 1$, and this occurs with probability at least $1-\delta / 2$.

By Eqs. (a6) and (a18) we see that with probability at least $\delta / 2$ both the sequence $c_{t}, t \geq \tau$ is bounded from below by $\varepsilon c>0$ and has a subsequence $c_{t_{k}}, k \geq 1$, converging to zero. This is impossible.

Let $\varepsilon=1$. There are two possibilities, namely, first, there is a sequence of finite time instants $t_{k}, k \geq 1$, such that $\lambda H\left(Q_{t_{k}}\right) / v C-m \geq d$, or, second, $\lambda H\left(Q_{t}\right) / v C-m<d$ starting from a finite time instant $\tau^{\prime \prime}$ with probability one.

In the first case by Eq. (a17) we see that for every firm coming at some $t_{k} \geq \max \left(t^{*}, \tau\right)$

$$
\begin{aligned}
\frac{c_{t_{k}+1}}{c_{t_{k}+1}^{\prime}}=\frac{c_{t_{k}}}{c_{t_{k}}^{\prime}}\left\{1-\frac{\lambda}{v C} \frac{m-m^{\prime}}{1-d+\frac{\lambda}{v C}\left[H\left(Q_{t_{k}}\right)-m^{\prime}\right]}\right\} \\
\quad \leq \frac{c_{t_{k}}}{c_{t_{k}}^{\prime}}\left\{1-\frac{\lambda}{v C} \frac{m-m^{\prime}}{1-d+\frac{\lambda}{v C}\left[H(0)-m^{\prime}\right]}\right\}=\frac{c_{t_{k}}}{c_{t_{k}}^{\prime}} r,
\end{aligned}
$$


where $c_{t_{k}}^{\prime}$ stands for the capital and $m^{\prime}$ for the variable costs of the newcomer. Since we are in the time domain where every entrant dies in a finite time, this firm dies at $t_{1}^{\prime}>t_{k}$. By Eqs. (a17) and (a20) we see that

$$
\frac{c_{t_{1}^{\prime}}}{c_{t_{1}^{\prime}}^{\prime}} \leq \frac{c_{t_{i}^{\prime}-1}}{c_{t_{1}^{\prime}-1}^{\prime}} \leq \cdots \leq \frac{c_{t_{k}+1}}{c_{t_{k}+1}^{\prime}} \leq r \frac{c_{t_{k}}}{c_{t_{k}}^{\prime}}
$$

Thus

$$
c_{t_{1}^{\prime}} \leq r c_{t_{k}} \frac{c_{t_{1}^{\prime}}^{\prime}}{c_{t_{k}}^{\prime}} \leq r c_{t_{k}} \frac{\varepsilon c}{c} \leq r c_{t_{k}}
$$

By Eq. (a19) we conclude that during the life time of any firm coming from $\max \left(t^{*}\right.$, $\tau$ ) onward, the firm living infinitely long at the very least does not gain anything in terms of capital stock. But it shrinks $r$ times during the life times of firms coming at $t_{k} \geq \max \left(t^{*}, \tau\right)$. This occurs with probability not less than $1-\delta / 2$. Thus, with the same probability there is a sequence $t_{k}^{\prime}, k \geq 1$, such that $c_{t^{\prime}} \rightarrow 0$ as $k \rightarrow \infty$. By Eqs. (a6) and (a18) we conclude that with probability at least $\delta / 2$ both the sequence $c_{t}$, $t \geq \tau$, is bounded from below by $\varepsilon c>0$ and has a subsequence $c_{t^{\prime}}, k \geq 1$, converging to zero. This is impossible.

Now let $\lambda H\left(Q_{t}\right) / v C-m<d$ starting from a finite time instant $\tau^{\prime \prime}$ with probability one. Hence, from $\tau^{\prime \prime}$ onward, the total productive capacity evolves in the domain where the notional firm surviving for infinite time shrinks. Consequently, we must have that $\lambda H\left(Q_{t}\right) / v C=d+m+o_{t}(1)$ where $o_{t}(1) \rightarrow 0$ with probability one as $t \rightarrow \infty$. This implies that with probability at least $1-\delta / 2$ every firm coming after $\max \left(t^{*}\right.$, $\left.\tau^{\prime \prime}\right)$ will be unboundedly growing (almost as $\left(1+m-m^{\prime}\right)^{t}$ for $\left.t \rightarrow \infty\right)$ in contradiction with the uniform boundness of the total productive capacity.

Thus we have showed that assuming that there is a firm surviving infinitely long with positive probability yields a contradiction.

The theorem is proved.

\section{References}

Baldwin, J.R., 1995. The Dynamics of Industrial Competition. Cambridge University Press, Cambridge. Carroll, G.R., 1997. Long-term evolutionary changes in organizational populations: theory, models and empirical findings. Indust. Corp. Change 6, 119-143.

Carroll, G.R., Hannan, M.T. (Eds.), 1995. Organizations in Industry. Oxford University Press, New York and Oxford.

Davis, S.T., Haltiwanger. J.C., Schuh, S., 1996. Job Creation and Destruction. MIT Press, Cambridge, MA.

Doob, J.L., 1953. Stochastic Processes. John Wiley, New York.

Dosi, G., 1988. Sources, procedures and microeconomic effects of innovation. J. Econ. Liter. 26 (3), $1120-1171$.

Dosi, G., Marsili, O., Orsenigo, L., Salvatore, R., 1995. Learning, market selection and the evolution of industrial structures. Small Bus. Econ. 7, 411-436.

Dunne, T., Roberts, M.J., Samuelson, L., 1988. Patterns of firm entry and exit in U.S. manufacturing industries. Rand J. Econ. 19, 495-515. 
Ericson, R., Pakes, A., 1995. Markov-perfect industry dynamics: a framework for empirical work. Rev. Econ. Stud. 62, 53-82.

Freeman, C., Soete, L., 1997. The Economics of Industrial Innovation, third ed. MIT Press, Cambridge, MA.

Geroski, P.A., 1995. What do we know about entry? Inter. J. Indust. Organ. 13, 421-440.

Geroski, P.A., Schwalbach, J. (Eds.), 1991. Entry and Market Contestability. An International Comparison. Basil Blackwell, Oxford.

Hannan, M.T., Freeman, J., 1989. Organizational Ecology. Harvard University Press, Cambridge, MA.

Hopenhayn, H.A., 1992. Entry, exit and firm dynamics in long run equilibrium. Econometrica 60, $1127-1150$.

Ijiri, Y., Simon, H.A., 1974. Interpretations of departures from the Pareto curve firm-size distributions. J. Polit. Econ. 82, 315-331.

Jovanovic, B., 1982. Selection and evolution of industry. Econometrica 50, 649-670.

Loève, M., 1955. Probability Theory. D. van Nostrand Company, New York.

Malerba, F., Orsenigo, L., 1995. Schumpeterian patterns of innovation. Cam. J. Econ. 19 (1), 47-65.

Schumpeter, J.A., 1934. Theory of Economic Development: An inquiry into Profits, Capital, Interest and the Business Cycle. Harvard University Press, Cambridge, MA.

Sutton, J., 1998. Technology and Market Structure. MIT Press, Cambridge, MA.

Valente, M., 1997. Laboratory for Simulation Development. User Manual, Interim Report IR-97-020/ May 1997, International Institute for Applied Systems Analysis, Laxenburg, Austria.

Winter, S.G., Kaniovski, Y.M., Dosi, G., 1997. A Baseline Model of Industry Evolution. Interim Report IR-97-013/March, International Institute for Applied Systems Analysis, Laxenburg, Austria. 


\section{Ordering Information}

Orders must include the publication number and should be sent to the Publications Department, International Institute for Applied Systems Analysis, A-2361 Laxenburg, Austria.

Telephone: +432236807

Telefax: +43223671313

E-mail: molina@iiasa.ac.at

A full list of IIASA publications is available at www.iiasa.ac.at 
International Institute for Applied Systems Analysis

Schlossplatz 1, A-2361 Laxenburg, Austria

$\rightarrow$ Tel: +432236807 Fax: +43223671313

II ASA www.iiasa.ac.at 